\title{
A Mixed Stress/Displacement Approach Model of Homogeneous Shells for Elastodynamic Problems
}

\author{
Axel Fernando Domínguez Alvarado iD and Alberto Díaz Díaz $(\mathbb{D}$ \\ Centro de Investigación en Materiales Avanzados S.C. Miguel de Cervantes 120, Complejo Industrial Chihuahua, \\ 31136 Chihuahua, Mexico \\ Correspondence should be addressed to Alberto Díaz Díaz; alberto.diaz@cimav.edu.mx
}

Received 4 September 2019; Accepted 11 December 2019; Published 13 January 2020

Academic Editor: George Tsiatas

Copyright @ 2020 Axel Fernando Domínguez Alvarado and Alberto Díaz Díaz. This is an open access article distributed under the Creative Commons Attribution License, which permits unrestricted use, distribution, and reproduction in any medium, provided the original work is properly cited.

\begin{abstract}
This paper presents the development of a model of homogeneous, moderately thick shells for elastodynamic problems. The model is obtained by adapting and modifying SAM-H model (stress approach model of homogeneous shells) developed by Domínguez Alvarado and Díaz in (2018) for static problems. In the dynamic version of SAM-H presented herein, displacements and stresses are approximated by polynomials of the out-of-plane coordinate. The stress approximation coincides with the static version of SAM-H when dynamic effects are neglected. The generalized forces and displacements appearing in the approximations are the same as those involved in a classical, moderately thick shell model (CS model) but the stress approximation adopted herein is more complex: the 3D motion equations and the stress boundary conditions at the faces of the shell are verified. The generalized motion and constitutive equations of dynamic SAM-H model are obtained by applying a variant of Euler-Lagrange equation which includes pertinently Hellinger-Reissner functional. In the constitutive equations, Poisson's effect of out-of-plane normal stresses on in-plane strains is not ignored; this is one important feature of SAM-H. To test the accuracy of dynamic SAM-H model, the following structures were considered: a hollow sphere and a catenoid. In each case, eigenfrequencies are first calculated and then a frequency analysis is performed applying a harmonic load. The results are compared to those of a CS model, MITC6 (mixed interpolation of tensorial components with 6 nodes per element) shell element calculations, and solid finite element computations. In the two problems, CS, MITC6, and dynamic SAM-H models yield accurate eigenfrequencies and eigenmodes. Nevertheless, the frequency analysis performed in each case showed that dynamic SAM-H provides much more accurate amplitudes of stresses and displacements than the CS model and the MITC6 shell finite element technique.
\end{abstract}

\section{Introduction}

Shell-like structures are a common solution for light and innovative designs; their structural analysis may be performed by making use of finite element calculations. The application of 3D solid elements requires high computational resources. Surface elements (2D elements) are an alternative to obtain accurate results for a reasonable computing cost [1].

Surface elements are based on shell models, which can be classified according to their ability to study thin $[2,3]$, moderately thick $[4,5]$, or thick shells $[6-8]$. The modelling in some shell models starts by proposing a displacement and/or stress field form in the thickness direction; in [9], Carrera classifies these models as axiomatic. In thin shell models, the displacement approach is similar to that in Kirchhoff-Love plate theory: 3 generalized displacements depending only on the in-plane coordinates are considered and the rotations are deduced from the generalized displacements and their spatial derivatives. In moderately thick shell models, the displacement approach is similar to that in Mindlin plate theory: 3 generalized displacements and 2 generalized rotations depending only on the in-plane coordinates are considered. Some examples of displacement approach models are Sanders model [10], the model built by Reddy in [5], and the Donnell-Mushtari-Vlasov (DMV) model [3]. Sanders shell theory derives six generalized equations of motion by means of the principle of virtual work. The model obtained in [5], hereinafter 
referred as to the classical shell model (CS model), is based on Hamilton's principle and has 5 generalized motion equations. In DMV theory, the generalized motion equations are reduced from 5 to 3 by neglecting some terms of transverse generalized forces and assuming symmetric in-plane generalized forces. In the three theories above, the applied loads at the inner and outer faces of the shell do not appear explicitly in the motion equations. Other inconvenience of these models is the neglection of Poisson's effect of out-ofplane normal stresses on in-plane strains; this can lead to an inaccurate displacement evaluation, particularly for thick and moderately thick shells [1]. Besides, the stress field deduced from the displacement approach models does not evaluate out-of-plane stresses accurately. In $[11,12]$, Tornabene and Viola used a displacement approach model and proposed a postprocessing technique (the differential quadrature method) for recovering and correcting the stress field in static problems. Another alternative to enhance the stress evaluation is the application of stress approach models; some examples of such models are those developed by Trefftz in $[13]$ and Voyiadjis in $[7,8]$. The Trefftz model does not verify the stress boundary conditions at the faces of the shell whilst the models proposed by Voyiadjis do verify these conditions and also the $3 \mathrm{D}$ equilibrium equations. To the best of our knowledge, in the literature, there are no pure stress approach models which are applied to dynamic problems and verify the $3 \mathrm{D}$ motion equations. Mixed approach models approximate both displacement and stress fields simultaneously $[6,14,15]$. Fang et al. developed in [6] the equations of their static mixed model by making use of the Hellinger-Reissner functional proposed in [16]. The stress field in their model fulfilled the stress boundary conditions at the faces of the shell but did not verify the $3 \mathrm{D}$ equilibrium equations.

In order to evaluate the accuracy of dynamic shell models, researchers usually calculate eigenfrequencies and eigenmodes [17-21]. The eigenmodes and eigenfrequencies are compared to those obtained by other models and solid finite elements. Convergence speed is often discussed. In [22], Leissa analyzed and compared several theories of thin shells such as those developed by Love [2], Sanders [10], Flügge [23], Naghdi [4], and Donnell-Mushtari-Vlasov (DMV) [3]. Several loading conditions, geometries, and thickness-to-radius of curvature ratios were considered. In some cases, a 3D exact solution was available, and the accuracy of the eigenvalues obtained by the different models was analyzed. The DMV model was the least accurate. In dynamic problems where a harmonic load is imposed, it is important to carry out a frequency domain analysis and to determine the amplitudes of displacements and stresses. However, it is less usual to find these analysis with shell models in the literature. In [24], Poultangari and NikkhahBahrami calculated the displacement amplitudes in thin cylinders given by the Flügge shell model [23] combined with an extended wave method and validated these results by comparing them with those obtained by solid finite elements.

The resolution of the equations of shell models can be performed by making use of the finite element method.
Many commercial finite element software packages include surface elements (shell elements). A well-known family of shell elements using a displacement approach model is the MITCn (mixed interpolation of tensorial components, where $n$ refers to the number of nodes in the element) [25]. The development of these elements is based on pertinent displacement and strain interpolations [26]; the stress-strain constitutive equation of the shell model yields the element rigidity matrix. These elements do not exhibit shear and membrane locking numerical problems which commonly appear in low-order elements [27].

In the present paper, a mixed stress/displacement approach model for elastodynamic problems of moderately thick, homogeneous shells is developed. The model is inspired by the stress approach model SAM-H for static shells proposed by Domínguez Alvarado and Díaz in [1]. This model proved to be very accurate in the evaluation of stresses since $3 \mathrm{D}$ equilibrium equations and boundary conditions at the faces of the shell are satisfied. The static SAM-H model equations were obtained by making use of the Hellinger-Reissner functional [16] of elastostatic problems. The generalized constitutive equations of the model take into account Poisson's effect of out-of-plane normal stresses on inplane strains, and this consideration provided higher accuracy to the model, particularly for thicker shells. The dynamic model developed in this paper coincides with the static SAM-H model when accelerations are neglected. For this reason, the name SAM-H is conserved and the new model is referred to as the dynamic SAM-H model. In the dynamic SAM-H model, 3D displacement and stress approximations are proposed. The latter approximation satisfies the stress boundary conditions at the faces of the shell and the $3 \mathrm{D}$ motion equation: this is the first originality of this study. The main goal is to provide an accurate estimation of the stress field. In order to obtain dynamic SAM-H equations, the EulerLagrange equation is applied. In this equation, the Hellinger-Reissner functional is used as a potential energy of the Lagrangian. The generalized motion and constitutive equations dynamic SAM-H involve explicitly the applied normal and shear stresses at the faces of the shell: this is a second originality of the model. As in the static version, another important feature of the dynamic model is the inclusion of Poisson's effect of out-ofplane normal stresses on in-plane strains. The model equations are then applied to the dynamic analysis of two pressurized structures: a hollow sphere and a catenoid. For each structure, eigenvalues/eigenfrequencies and amplitudes of stresses/displacements in case of a forced vibration are compared to those obtained with solid finite elements, CS model, and MITC6 surface elements.

This article is organized as follows. In Section 2, the Hellinger-Reissner functional and Euler-Lagrange equation are recalled and combined to establish the method to derive the equations of the shell model. In Section 3, the Euler-Lagrange equation is applied to obtain dynamic SAM-H equations. In Section 4, two application examples are shown and discussed to validate 
the model equations. Section 5 contains the main conclusions of this study.

\section{Hellinger-Reissner Functional Combined with Euler-Lagrange Equation for Shell Modelling}

A homogeneous, doubly curved shell lying in the volume $\Omega$ and delimited by the boundary $\partial \Omega$ is considered. Its thickness $h$ is uniform, and $\omega$ denotes its middle surface. The principal curvatures are $\kappa_{1}$ and $\kappa_{2}$. Orthogonal curvilinear coordinates $\left(\xi_{1}, \xi_{2}, \xi_{3}\right)$ are adopted; the unit vectors in the directions of the lines of curvature are $\mathbf{e}_{1}$ and $\mathbf{e}_{2}$ whilst the unit vector $\mathbf{e}_{3}$ normal to the middle surface is obtained by the cross product $\mathbf{e}_{1} \times \mathbf{e}_{2}$. The surface metric tensor components are $g_{\alpha \beta}(\alpha, \beta=1,2)$, and the following coefficients are defined: $a_{1}=\sqrt{g_{11}}$ and $a_{2}=\sqrt{g_{22}}$. The material is orthotropic and one of its orthotropy directions is direction 3 .

In what follows, the following assumptions and notations are adopted:

(i) $3 \mathrm{D}$ vectors and tensors are written with bold face characters

(ii) First- and second-order tensors of the $2 \mathrm{D}$ space (defined by the basis $\left(\mathbf{e}_{1}, \mathbf{e}_{2}\right)$ ) are noted in bold underlined and double underlined characters, respectively

(iii) $\dot{r}$ and $\ddot{r}$ denote first and second time derivatives of field $r$

(iv) Greek subscripts take the values 1 and 2

(v) The outer $\left(\xi_{3}=h / 2\right)$ and inner $\left(\xi_{3}=-h / 2\right)$ faces of the shell are subjected to the stress vectors $\mathbf{s}^{+}=$ $\underline{\tau}^{+}+\sigma^{+} \mathbf{e}_{3}$ and $\mathbf{s}^{-}=\underline{\tau}^{-}+\sigma^{-} \mathbf{e}_{3}$, respectively; $\underline{\tau}^{+}$ and $\tau^{-}$are the applied shear stress vectors; and $\sigma^{+}$ and $\sigma^{-}$are the applied normal stresses

(vi) A $3 \mathrm{D}$ body load vector $\mathbf{f}=f_{1} \mathbf{e}_{1}+f_{2} \mathbf{e}_{2}+f_{3} \mathbf{e}_{3}$, uniform through the thickness direction, is applied; it is worth defining the in-plane body load vector $\underline{f}=f_{1} \mathbf{e}_{1}+f_{2} \mathbf{e}_{2}$

(vii) The curvatures $\kappa_{1}$ and $\kappa_{2}$ vary smoothly, in such a manner that $\partial \kappa_{\beta} / \partial \xi_{\alpha} \simeq 0$

(viii) Any term multiplied by $\left(h \kappa_{\alpha}\right)^{n}$ is neglected if $n \geq 2$

2.1. Hellinger-Reissner Functional of Elastostatic Problems. On the boundaries $\partial \Omega_{\mathbf{u}}$ and $\partial \Omega_{\mathbf{s}}$ of $\Omega$, the body is subjected to the displacement vector $\mathbf{u}^{g}$ and stress vector $\mathbf{s}^{g}$, respectively. The Hellinger-Reissner functional for elastic problems [16] applied to the shell is

$$
\begin{aligned}
\text { H.R. }\left(\mathbf{u}^{*}, \boldsymbol{\sigma}^{*}\right)= & \int_{\Omega}\left[\boldsymbol{\sigma}^{*}: \boldsymbol{\varepsilon}\left(\mathbf{u}^{*}\right)-\mathbf{f} \cdot \mathbf{u}^{*}-w_{e}\left(\boldsymbol{\sigma}^{*}\right)\right] d \Omega \\
& -\int_{\partial \Omega_{\mathrm{u}}}\left(\boldsymbol{\sigma}^{*} \cdot \mathbf{n}\right) \cdot\left(\mathbf{u}^{*}-\mathbf{u}^{g}\right) \mathrm{d} S-\int_{\partial \Omega_{\mathrm{s}}} \mathbf{s}^{g} \cdot \mathbf{u}^{*} \mathrm{~d} S,
\end{aligned}
$$

where - and : stand for simple and double tensor contractions, respectively; $\mathbf{n}$ is the $3 \mathrm{D}$ outward-pointing normal vector to the boundary of $\Omega$; $\mathbf{u}^{*}$ is a piecewise $\mathrm{C} 1$ first-order tensor field; $\boldsymbol{\sigma}^{*}$ is a piecewise $\mathrm{C} 1$ second-order symmetric tensor field; $\boldsymbol{\varepsilon}$ is Cauchy's infinitesimal strain tensor; $w_{e}\left(\boldsymbol{\sigma}^{*}\right)=(1 / 2) \boldsymbol{\sigma}^{*}: \mathbf{S}: \boldsymbol{\sigma}^{*}$ is the elastic energy density; and $\mathbf{S}$ is the $3 \mathrm{D}$, fourth-order compliance tensor. In what follows, a field with an uppercase ${ }^{*}$ denotes a field that is not necessarily the solution of the problem and varies in its corresponding virtual space. An integration by parts for the term containing $\boldsymbol{\sigma}^{*}: \boldsymbol{\varepsilon}\left(\mathbf{u}^{*}\right)$ yields an alternative form of the Hellinger-Reissner functional:

$$
\begin{aligned}
\text { H.R. }\left(\mathbf{u}^{*}, \boldsymbol{\sigma}^{*}\right)= & -\int_{\Omega}\left[\operatorname{div} \boldsymbol{\sigma}^{*} \cdot \mathbf{u}^{*}+\mathbf{f} \cdot \mathbf{u}^{*}+w_{e}\left(\boldsymbol{\sigma}^{*}\right)\right] d \Omega \\
& +\int_{\partial \Omega_{\mathbf{u}}}\left(\boldsymbol{\sigma}^{*} \cdot \mathbf{n}\right) \cdot \mathbf{u}^{g} \mathrm{~d} S+\int_{\partial \Omega_{\mathbf{s}}}\left(\boldsymbol{\sigma}^{*} \cdot \mathbf{n}-\mathbf{s}^{g}\right) \cdot \mathbf{u}^{*} \mathrm{~d} S,
\end{aligned}
$$

where div is the divergence operator in the $3 \mathrm{D}$ space. Reissner's theorem [16] states that

(i) The solution of the mechanical problem is the couple $(\mathbf{u}, \boldsymbol{\sigma})$ which makes the H.R. functional stationary (notice that no uppercase ${ }^{*}$ appears for the solution)

(ii) The stationarity of H.R. with respect to $\mathbf{u}^{*}$ yields the equilibrium equations and the boundary conditions on the stress vector whilst its stationarity with respect to $\boldsymbol{\sigma}^{*}$ provides the constitutive equations and the boundary conditions on the displacement field

2.2. Euler-Lagrange Equation. In Lagrangian mechanics, the evolution of a dynamic system is obtained by solving the Euler-Lagrange equation:

$$
\frac{\mathrm{d}}{\mathrm{d} t}\left(\frac{\partial \mathscr{L}}{\partial \dot{q}_{i}}\right)-\frac{\partial \mathscr{L}}{\partial q_{i}}=0 .
$$

$\mathscr{L}$ is called the Lagrangian and is defined by

$$
\mathscr{L}\left(q_{i}, \dot{q}_{i}\right)=K\left(\dot{q}_{i}\right)-\Pi\left(q_{i}\right),
$$

where $q_{i}$ are the generalized coordinates $(1 \leq i \leq n, n$ is the number of generalized coordinates) and $K$ and $\Pi$ are the kinetic and potential energies, respectively. In this work, $\Pi$ is considered as the Hellinger-Reissner functional defined in equation (1). In linear elastic problems, the generalized coordinates $q_{i}$ are the components of the stress and displacement fields: $\boldsymbol{\sigma}^{*}$ and $\mathbf{u}^{*}$. The expression of the kinetic energy is

$$
K\left(\dot{\mathbf{u}}^{*}\right)=\frac{1}{2} \int_{\Omega} \rho \dot{\mathbf{u}}^{*} \cdot \dot{\mathbf{u}}^{*} d \Omega,
$$

where $\rho$ is the volumetric mass density of the material. Then, the Euler-Lagrange equation (3) yields 


$$
\begin{aligned}
\frac{\mathrm{d}}{\mathrm{d} t}\left(\frac{\partial K}{\partial \dot{\mathbf{u}}^{*}}\right)+\frac{\partial \mathrm{HR}}{\partial \mathbf{u}^{*}} & =0, \\
\frac{\partial \mathrm{HR}}{\partial \boldsymbol{\sigma}^{*}} & =0 .
\end{aligned}
$$

The couple $(\mathbf{u}, \boldsymbol{\sigma})$ that satisfies equations (6) and (7) is the solution of the problem. From equation (6), the $3 \mathrm{D}$ motion equations and stress boundary conditions are obtained. From equation (7), one deduces the 3D constitutive equations and the displacement boundary conditions.

2.3. Case of a Shell Model Based on a Mixed Approach. When dealing with plates or shells, mixed-approach models provide an approximation of the $3 \mathrm{D}$ stress and displacement fields. In this approximation, polynomials of the coordinate across the thickness are usually applied. The coefficients of these polynomials involve the generalized forces $\left(\mathbf{F}^{G}\right)$ and displacements $\left(\mathbf{U}^{G}\right)$. These generalized fields depend only on the in-plane coordinates $\left(\xi_{1}, \xi_{2}\right)$. The stress approximation may be chosen in order to verify the $3 \mathrm{D}$ stress boundary conditions at the faces of the shell and/or to obtain a good estimation of the 3D motion equations.

The Euler-Lagrange equation can be applied to derive the generalized equations of mixed-approach models. The generalized coordinates are $\mathbf{F}^{G}$ and $\mathbf{U}^{G}$. The Euler-Lagrange equation yields

$$
\begin{aligned}
\frac{\mathrm{d}}{\mathrm{d} t}\left(\frac{\partial K}{\partial \dot{\mathbf{U}}^{G *}}\right)+\frac{\partial \mathrm{HR}}{\partial \mathbf{U}^{G *}} & =0, \\
\frac{\partial \mathrm{HR}}{\partial \mathbf{F}^{G *}} & =0 .
\end{aligned}
$$

The generalized motion equations and force edge conditions are obtained from equation (8). The generalized constitutive equations and displacement edge conditions are given by equation (9).

\section{Dynamic SAM-H Model}

SAM-H (stress approach model of homogeneous shells) is a model originally developed for static problems of moderately thick shells [1]. Herein, this model is adapted and modified so as to enable the resolution of dynamic problems. Basically, the modification consists in adding an approximation of the $3 \mathrm{D}$ displacement field in order to use the methodology shown in Section 2.3. A new model including accelerations in the motion equations is obtained. When dynamic effects are neglected, the new model equations are the same as those of the static SAM-H equations developed in [1]. It is for this reason that we decided to preserve the name $\mathrm{SAM}-\mathrm{H}$ for the new model even if it is based on a mixed approach: the new model will be referred to as the dynamic SAM-H model.
3.1. Approximation of Stress and Displacement Fields. For this approximation, the following polynomial basis of 4-th degree $\xi_{3}$-polynomials is defined:

$$
\begin{aligned}
& P_{0}\left(\xi_{3}\right)=1 \\
& P_{1}\left(\xi_{3}\right)=\frac{\xi_{3}}{h} \\
& P_{2}\left(\xi_{3}\right)=-6\left(\frac{\xi_{3}}{h}\right)^{2}+\frac{1}{2} \\
& P_{3}\left(\xi_{3}\right)=-2\left(\frac{\xi_{3}}{h}\right)^{3}+\frac{3}{10}\left(\frac{\xi_{3}}{h}\right) \\
& P_{4}\left(\xi_{3}\right)=-\frac{14}{3}\left(\frac{\xi_{3}}{h}\right)^{4}+\left(\frac{\xi_{3}}{h}\right)^{2}-\frac{1}{40} .
\end{aligned}
$$

The displacement approximation is chosen as follows:

$$
\mathbf{u}^{*}=\left(\begin{array}{c}
U_{1}^{*}\left(\xi_{1}, \xi_{2}, t\right) P_{0}\left(\xi_{3}\right)+h \phi_{1}\left(\xi_{1}, \xi_{2}, t\right) P_{1}\left(\xi_{3}\right) \\
U_{2}^{*}\left(\xi_{1}, \xi_{2}, t\right) P_{0}\left(\xi_{3}\right)+h \phi_{2}\left(\xi_{1}, \xi_{2}, t\right) P_{1}\left(\xi_{3}\right) \\
U_{3}^{*}\left(\xi_{1}, \xi_{2}, t\right) P_{0}\left(\xi_{3}\right)
\end{array}\right),
$$

where $U_{1}, U_{2}, U_{3}, \phi_{1}$, and $\phi_{2}$ are the generalized displacements.

The generalized forces of the model are membrane forces $\underline{\mathbf{N}}^{*}$, shear forces $Q^{*}$, and moments $\underline{\mathbf{M}}^{*}$; their components $\overline{\overline{a r e}}$ defined as follows:

$$
\begin{aligned}
N_{\alpha \beta}^{*} & =\int_{-(h / 2)}^{h / 2} \sigma_{\alpha \beta}^{*}\left(1+\frac{\xi_{3}}{R_{3-\beta}}\right) d \xi_{3}, \\
Q_{\alpha}^{*} & =\int_{-(h / 2)}^{h / 2} \sigma_{\alpha 3}^{*}\left(1+\frac{\xi_{3}}{R_{3-\alpha}}\right) d \xi_{3}, \\
M_{\alpha \beta}^{*} & =\int_{-(h / 2)}^{h / 2} \xi_{3} \sigma_{\alpha \beta}^{*}\left(1+\frac{\xi_{3}}{R_{3-\beta}}\right) d \xi_{3},
\end{aligned}
$$

where $R_{1}$ and $R_{2}$ are the principal radii of curvature; the subscript $3-\alpha$ is 2 if $\alpha=1$, and 1 if $\alpha=2$. The same can be said for the subscript $3-\beta$. The stress tensor components are approximated by

$$
\begin{aligned}
& \sigma_{\alpha \beta}^{*}\left(\xi_{1}, \xi_{2}, \xi_{3}, t\right)=\sum_{n=0}^{1} \sigma_{\alpha \beta}^{n *}\left(\xi_{1}, \xi_{2}, t\right) P_{n}\left(\xi_{3}\right), \\
& \sigma_{\alpha 3}^{*}\left(\xi_{1}, \xi_{2}, \xi_{3}, t\right)=\sum_{n=0}^{3} \sigma_{\alpha 3}^{n *}\left(\xi_{1}, \xi_{2}, t\right) P_{n}\left(\xi_{3}\right), \\
& \sigma_{33}^{*}\left(\xi_{1}, \xi_{2}, \xi_{3}, t\right)=\sum_{n=0}^{4} \sigma_{33}^{n *}\left(\xi_{1}, \xi_{2}, t\right) P_{n}\left(\xi_{3}\right),
\end{aligned}
$$

where $\sigma_{i j}^{n *}(1 \leq i, j \leq 3)$ are stress coefficients which are expressed as linear combinations of the generalized forces. The polynomial degree of each stress component and the 
coefficients $\sigma_{i j}^{n *}$ were chosen so as to verify the 3D stress boundary conditions at the faces of the shell and the $3 \mathrm{D}$ motion equation:

$$
\operatorname{div} \sigma^{*}+\mathbf{f}=\rho \ddot{\mathbf{u}}^{*} .
$$
shown.

In Appendix A, the expressions of the coefficients $\sigma_{i j}^{n *}$ are

It is important to note that the displacement approximation is the same as that in the CS model. The generalized forces are the same in SAM-H and CS models. Nevertheless, the CS model obtains the 3D stress field from the $3 \mathrm{D}$ constitutive equations which require the calculation of the $3 \mathrm{D}$ strains by making use of the displacement approximation. It is for this reason that the $3 \mathrm{D}$ stress field in the CS model does not verify the $3 \mathrm{D}$ motion equations and the boundary conditions at the faces of the shell.

\subsection{Generalized Motion Equations and Boundary Conditions} on Generalized Forces. After introducing the 3D stress and displacement approximations in equation (2) and applying equation (8), one identifies the following generalized motion equations:

$$
\begin{gathered}
\underline{\operatorname{div}} \underline{\underline{\mathbf{N}}}+\underline{\underline{\kappa}} \cdot \underline{Q}+\frac{h\left(\kappa_{1}+\kappa_{2}\right)}{2}\left(\underline{\tau}^{+}-\underline{\tau}^{-}\right)+\underline{\tau}^{+}+\underline{\tau}^{-}+h f=\rho h \underline{\mathbf{U}}+\frac{\rho h^{3}\left(\kappa_{1}+\kappa_{2}\right)}{12} \ddot{\boldsymbol{\phi}}, \\
\operatorname{div} \underline{\mathbf{Q}}+\left(\sigma^{+}-\sigma^{-}\right) \frac{h\left(\kappa_{1}+\kappa_{2}\right)}{2}+\sigma^{+}+\sigma^{-}-N_{11} \kappa_{1}-N_{22} \kappa_{2}+h f_{3}=\rho h \ddot{U}_{3}, \\
\underline{\operatorname{div}} \underline{\underline{\mathbf{M}}-Q}+\frac{h^{2}\left(\kappa_{1}+\kappa_{2}\right)}{4}\left(\underline{\boldsymbol{\tau}}^{+}+\underline{\boldsymbol{\tau}}^{-}\right)+\frac{h}{2}\left(\underline{\boldsymbol{\tau}}^{+}-\underline{\boldsymbol{\tau}}^{-}\right)+\frac{h^{3}\left(\kappa_{1}+\kappa_{2}\right)}{12} \underline{\mathbf{f}}=\frac{\rho h^{3}\left(\kappa_{1}+\kappa_{2}\right)}{12} \underline{\mathbf{\mathbf { U }}}+\frac{\rho h^{3}}{12} \ddot{\boldsymbol{\phi}},
\end{gathered}
$$

where $\underline{\boldsymbol{\kappa}}=\kappa_{1} \mathbf{e}_{1} \otimes \mathbf{e}_{1}+\kappa_{2} \mathbf{e}_{2} \otimes \mathbf{e}_{2}$, in which $\otimes$ stands for the tensor product operation. In the generalized motion equations above, $\underline{\text { div }}$ and div stand for the divergences of second order tensor and vector fields in the $2 \mathrm{D}$ space of coordinates $\left(\xi_{1}, \xi_{2}\right)$, respectively. If the accelerations were neglected in these equations, one would obtain the equilibrium equations of static SAM-H model developed in [1]. Let us mention that these generalized motion equations are similar to those in the CS model except for the applied stresses at the faces of the shell which do not appear in the equations of the CS model.

Equation (8) also provides the edge conditions on the generalized forces:

$$
\begin{aligned}
& \underline{\underline{\mathbf{N}}} \cdot \underline{n}^{0}=\underline{F}^{g}, \\
& \underline{Q} \cdot \underline{n}^{0}=F_{3}^{g}, \\
& \underline{\underline{\mathbf{M}}} \cdot \underline{n}^{0}=\underline{C}^{g},
\end{aligned}
$$

where $\underline{n}^{0}=n_{1}^{0} \mathbf{e}_{1}+n_{2}^{0} \mathbf{e}_{2}$ is the outward-pointing normal vector to the edge of the middle surface $\omega$ and $\underline{F}^{g}, F_{3}^{g}$, and $\underline{C}^{g}$ are the given membrane force vector, shear force, and bending moment vector at the shell edges, respectively. The edge forces and bending moments shown in equation (20) are deduced from the applied $3 \mathrm{D}$ stress vector $\mathbf{s}^{g}$ at the boundary of the solid as follows:

$$
\left\{\begin{array}{l}
F_{i}^{g}=\int_{-(h / 2)}^{h / 2}\left[P_{0}+h\left(\left(n_{1}^{0}\right)^{2} \kappa_{1}+\left(n_{2}^{0}\right)^{2} \kappa_{2}\right) P_{1}\right] s_{i}^{g} d \xi_{3} \\
C_{\alpha}^{g}=\int_{-(h / 2)}^{h / 2} \frac{h^{2}}{12}\left(\left(n_{1}^{0}\right)^{2} \kappa_{1}+\left(n_{2}^{0}\right)^{2} \kappa_{2}\right) P_{0}\left(\xi_{3}\right) s_{\alpha}^{g} d \xi_{3}+\int_{-(h / 2)}^{h / 2} h P_{1}\left(\xi_{3}\right)\left[1-\frac{h}{6}\left(\left(n_{1}^{0}\right)^{2} \kappa_{1}+\left(n_{2}^{0}\right)^{2} \kappa_{2}\right)\right] s_{\alpha}^{g} d \xi_{3} .
\end{array}\right.
$$

\subsection{Generalized Constitutive Equations and Boundary Con-} ditions on Generalized Displacements. By introducing the stress and displacement approximation in the HellingerReissner functional in equation (1), one identifies the following generalized strain tensors:

$$
\begin{aligned}
& \underline{\underline{\varepsilon}}^{*}=\underline{\underline{\operatorname{grad}}} \underline{U}^{*}+U_{3}^{*} \underline{\underline{\kappa}}, \\
& \underline{\underline{\chi}}^{*}=\underline{\underline{\operatorname{grad}}} \underline{\phi}^{*}, \\
& \stackrel{\underline{\mathbf{d}}}{=} \underline{\underline{\underline{\operatorname{grad}}}} U_{3}^{*}+\underline{\phi}^{*}-\underline{\underline{\kappa}} \cdot \underline{\mathbf{U}},
\end{aligned}
$$


where $\quad \underline{\mathbf{U}}^{*}=U_{1}^{*} \mathbf{e}_{1}+U_{2}^{*} \mathbf{e}_{2}, \quad \phi^{*}=\phi_{1}^{*} \mathbf{e}_{1}+\phi_{2}^{*} \mathbf{e}_{2}, \quad$ and $\underline{\underline{\kappa}}^{\prime}=\kappa_{2} \mathbf{e}_{1} \otimes \mathbf{e}_{1}+\kappa_{1} \mathbf{e}_{2} \otimes \mathbf{e}_{2}$ in which $\otimes$ stands for the tensor product operation. The operators grad and grad are $2 \mathrm{D}$ gradients of vector and scalar fields, respectively.

After introducing the 3D stress and displacement approximations in equation (2) and applying equation (9), one obtains the generalized constitutive equations. So as to ease the derivation of these equations, the expression of the elastic energy density $w_{e}\left(\boldsymbol{\sigma}^{*}\right)=(1 / 2) \boldsymbol{\sigma}^{*}: \mathbf{S}: \boldsymbol{\sigma}^{*}$ is simplified as explained in Appendix B. In order to condense the obtained constitutive equations, the following vectors of generalized forces and strains are defined:

$$
\begin{aligned}
\mathbf{q} & =\left(\begin{array}{l}
\widehat{\mathbf{N}} \\
\hat{\mathbf{M}}
\end{array}\right), \\
\boldsymbol{\varepsilon} & =\left(\begin{array}{l}
\widehat{\mathcal{\varepsilon}} \\
\hat{\chi}
\end{array}\right), \\
\underline{\mathbf{d}} & =\left(\begin{array}{l}
d_{1} \\
d_{2}
\end{array}\right),
\end{aligned}
$$

where

$$
\begin{aligned}
\widehat{\mathbf{N}} & =\left(\begin{array}{l}
N_{11} \\
N_{22} \\
N_{12} \\
N_{21}
\end{array}\right), \\
\widehat{\mathbf{M}} & =\left(\begin{array}{l}
M_{11} \\
M_{22} \\
M_{12} \\
M_{21}
\end{array}\right), \\
\widehat{\boldsymbol{\varepsilon}} & =\left(\begin{array}{l}
\varepsilon_{11} \\
\varepsilon_{22} \\
\varepsilon_{12} \\
\varepsilon_{21}
\end{array}\right), \\
& \\
\chi \chi & =\left(\begin{array}{l}
\chi_{11} \\
\chi_{22} \\
\chi_{12} \\
\chi_{21}
\end{array}\right) .
\end{aligned}
$$

The constitutive equations are the same as those obtained in [1] for the static version of the model; they are written as follows:

$$
\left\{\begin{array}{l}
\mathbf{q}=\mathbf{K} \boldsymbol{\varepsilon}+\sigma^{+} \mathbf{k}^{+}+\sigma^{-} \mathbf{k}^{-}+f_{3} \mathbf{k}^{3}, \\
\underline{Q}=\mathbf{L} \underline{\mathbf{d}}+\mathbf{L}^{+} \underline{\tau}^{+}+\mathbf{L}^{-} \underline{\tau}^{-},
\end{array}\right.
$$

where $\mathbf{K}$ is an $8 \times 8$ stiffness matrix, $\mathbf{k}^{+}, \mathbf{k}^{-}$, and $\mathbf{k}^{3}$ are $8^{-}$ component stiffness vectors, and $\mathbf{L}, \mathbf{L}^{+}$, and $\mathbf{L}^{-}$are $2 \times 2$ stiffness matrices. The expressions of these matrices are shown in Appendix C. As already mentioned in [1], for the static SAM-H model, the consideration of $\mathbf{k}^{+}, \mathbf{k}^{-}, \mathbf{k}^{3}, \mathbf{L}^{+}$, and $\mathbf{L}^{-}$ represents an important originality which provides significant accuracy to SAM-H model, particularly when dealing with moderately thick shells. The terms $\mathbf{k}^{+}$and $\mathbf{k}^{-}$are related to the consideration of Poisson's effect of out-of-plane normal stresses on in-plane strains. Let us note that the form of these generalized constitutive equations is similar to that in the CS model. The expressions of the terms in the stiffness matrices are not the same in SAM-H and CS models. Another important difference is that the applied stresses at the shell faces and the volumetric force $f_{3}$ are not taken into account in the constitutive equations of the CS model. In fact, Poisson's effect of out-of-plane normal stresses on in-plane strains is neglected in the CS model as in most plate and shell models.

Equation (9) also provides the following edge conditions on the generalized displacement fields:

$$
\begin{aligned}
\underline{\mathbf{U}} & =\underline{\mathbf{U}}^{g}, \\
U_{3} & =U_{3}^{g}, \\
\underline{\phi} & =\underline{\phi}^{g},
\end{aligned}
$$

where $\underline{\mathrm{U}}^{g}, U_{3}^{g}$, and $\phi^{g}$ are the given in-plane displacement vector, out-of-plane displacement, and rotation vector at the shell edges, respectively.

\section{Results and Discussion}

In this section, SAM-H is applied to the resolution of two structural dynamic problems. In the first problem, an analytical resolution of the model equations is performed. In the other problem, SAM-H equations are solved numerically by making use of a commercial finite element software: COMSOL Multiphysics (5.3a version). In this software, the PDE physics interface is applied and the equations are implemented manually in a coefficient form. So as to validate the SAM-H model, its results are compared to those obtained with solid finite element calculations, the CS model developed in [5], and MITC6 shell finite elements [25] included in COMSOL Multiphysics.

4.1. Analytical Results for a Pressurized Hollow Sphere. For an analytical resolution of the SAM-H dynamic equations, a hollow sphere subjected to a harmonic internal pressure is considered; the external face of the sphere is stress free. The radius of the middle surface and the thickness-to-radius ratio are denoted by $R$ and $\eta$, respectively. The material is isotropic and characterized by its Young's modulus $E$, Poisson's ratio $\nu$, and density $\rho$. The internal pressure $p$ is given by $p(t)=p_{0} \sin (2 \pi \bar{f} t)$, where $p_{0}$ and $\bar{f}$ are the amplitude of the internal pressure and the excitation frequency, respectively. SAM-H results are solved analytically and compared to those obtained with the classical shell model (CS) and solid finite elements. Owing to a spherical symmetry (only radial displacements are allowed), the $3 \mathrm{D}$ solid problem can be reduced to a $1 \mathrm{D}$ problem (the spatial coordinate is the radial position) and the shell models are reduced to a $0 \mathrm{D}$ problem. The solid problem is solved by making use of COMSOL Multiphysics software. 
Let us start by analyzing the eigenfrequencies of the vibration modes that are excited by such a load. SAM-H and CS models yield only one vibration mode; the eigenfrequencies are identical for both models:

$$
f^{\mathrm{SAM}-\mathrm{H}}=f^{\mathrm{CS}}=\frac{1}{2 \pi} \sqrt{\frac{2 E}{\rho R^{2}(1-\nu)}} .
$$

The number of eigenfrequencies in the solid problem is infinite. The first eigenfrequency $f_{1}$ is related to a vibration mode analogue to those obtained by the shell models. The other eigenfrequencies $f_{n}(n \geq 2)$ are related to vibrations which are not reproduced by the shell models due to the simplicity of the adopted expression for the kinematic field $u_{3}$ (see equation (11)). For the particular problem studied herein and considering a moderately thick shell $(\eta \leq 0.3)$, the determination of the fundamental eigenfrequency and its corresponding vibration mode is enough to study the dynamic response of the structure. The relative error in the calculation of the fundamental eigenfrequency with SAM-H and CS models is defined by

$$
\delta f_{1}=\frac{f^{\mathrm{SAM}-\mathrm{H}}-f_{1}}{f_{1}} .
$$

In Figure 1(a), this relative error is plotted against the thickness to radius ratio $\eta$ for 5 values of Poisson's ratio $(-0.4,-0.2,0,0.2$, and 0.4$)$. It can be seen that for moderately thick shells, the predicted eigenfrequency by both shell models (SAM-H and CS models) is very accurate: the relative error $\delta f_{1}$ is less than $1.5 \%$. It is worth mentioning that neither Young's modulus nor the density affects the curves obtained in Figure 1(a).

Let us now make a frequency analysis applying a harmonic pressure. Transient vibrations are not calculated, and only the forced, steady-state vibrations are analyzed. The radial displacement amplitude given by the CS model is

$$
U_{3}^{\mathrm{CS}}=\frac{p_{0} R}{\eta}\left[\frac{1-\nu}{2 E-\rho \bar{\omega}^{2} R^{2}(1-\nu)}\right],
$$

where $\bar{\omega}=2 \pi \bar{f}$ is the excitation angular frequency. SAM-H model yields the following displacement amplitude:

$$
U_{3}^{\mathrm{SAM}}=k^{\mathrm{SAM}} \times U_{3}^{\mathrm{CS}} \text {, }
$$

where $k^{\mathrm{SAM}}$ can be considered as a correction factor appearing in SAM-H model to improve the determination of the displacement amplitude; the expression of $k^{\mathrm{SAM}}$ is given by

$$
\begin{aligned}
k^{\mathrm{SAM}} & =1-\eta-q^{-}, \\
q^{-} & =2 \eta \frac{45 \eta-1050 \nu+865 \eta \nu-1414 \eta v^{2}+1050 v^{2}}{2100(1-\nu)^{2}} .
\end{aligned}
$$

It is worth mentioning that the second term $(-\eta)$ appearing in the expression of $k^{\mathrm{SAM}}$ comes from a contribution of the normal stress $\sigma^{-}$in the motion equation (18) whilst the term $q^{-}$comes from the constitutive equations owing to Poisson's effect of out-of-plane normal stresses on in-plane strains. In Figure 1(b), the correction factor $k^{\text {SAM }}$ is plotted against the thickness to radius ratio $\eta$ for 5 values of Poisson's ratio $(-0.4,-0.2,0,0.2$, and 0.4$)$. One can observe that for very thin shells $(\eta \leq 0.01)$, no amendment is performed by SAM-H $\left(k^{\mathrm{SAM}} \simeq 1\right)$, but for thicker shells, the correction factor has to be taken into account, particularly for a negative Poisson's ratio.

Let us consider the following parameters and properties: $R=1 \mathrm{~m}, \quad \eta=0.3, \rho=1000 \mathrm{~kg} / \mathrm{m}^{3}, \quad E=1 \mathrm{GPa}$, $v=-0.4$, and $p_{0}=1 \mathrm{kPa}$. As shown in Figure $1(\mathrm{~b})$, it corresponds to the case where the largest differences between SAM-H and CS models are expected. In Figure 2(a), the amplitudes of the displacements at the middle surface provided by these models and solid finite elements (SFE) are plotted against the excitation frequency. The selected range of the excitation frequency includes only the first eigenfrequency of the sphere. At $130 \mathrm{~Hz}$, as compared to SFE, the relative errors in the displacement amplitude are $-5.1 \%$ and $49.5 \%$ for SAM-H and CS models, respectively. It can be seen that SAM-H makes a better approximation of the displacement amplitude than the CS model. The displacement amplitude peak of the shell models occurs at a $190 \mathrm{~Hz}$ frequency. In structural design, for safety reasons, it is a common practice to avoid resonance effects by ensuring that the fundamental eigenfrequency is greater enough than the excitation frequency. Let us assume that the excitation frequencies are at least $20 \%$ less than the fundamental eigenfrequency calculated by SAM-H. In Figure 2(b), the amplitude of the in-plane stresses $\sigma_{11}$ calculated at the middle surface by SAM-H, CS, and SFE techniques is plotted against the excitation frequency $\bar{f}$. In this figure, the curves of the out-of-plane normal stresses $\sigma_{33}$ evaluated at the middle surface by SAM-H and SFE are also included. Let us recall that the CS model is unable to evaluate this stress. It can be seen that within the range of excitation frequencies, SAM-H results of $\sigma_{11}$ are more accurate than those of the CS model and the evaluation of $\sigma_{33}$ by SAM-H at the middle surface is also accurate. Let us plot in Figure 3 the normal stress $\sigma_{33}$ as a function of the position $\xi_{3}$ across the thickness for two excitation frequencies: 50 and $150 \mathrm{~Hz}$.

The case of a pressurized sphere has shown that SAM-H and CS provide the same prediction of the fundamental eigenfrequency, and the value is very accurate. Nevertheless, SAM-H provides a much better approximation of displacement and stress field amplitudes in a frequency analysis.

4.2. Numerical Results for a Catenoid. In this section, a $0.9 \mathrm{~m}$ thick catenoid is considered; its middle surface is shown in Figure 4(a) and is defined by the following Cartesian coordinates:

$$
\begin{aligned}
x & =a \cosh (s) \cos (\phi), \\
x y & =-a \cosh (s) \sin (\phi), \\
z & =\text { as, }
\end{aligned}
$$




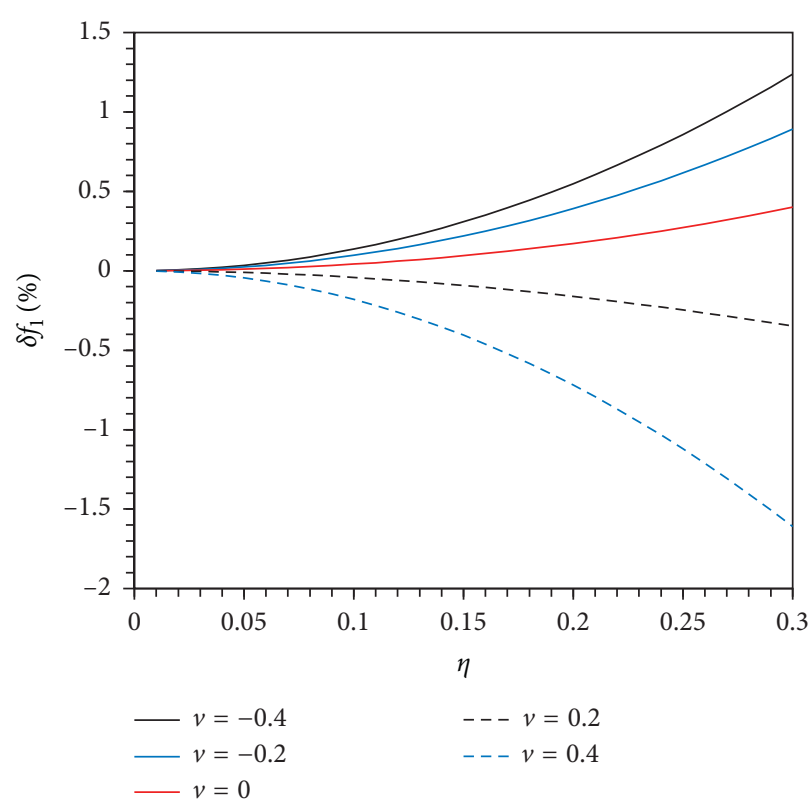

(a)



(b)

Figure 1: Relative error $\delta f_{1}$ vs. thickness to radius ratio (a) and correction factor vs. thickness to radius ratio (b).

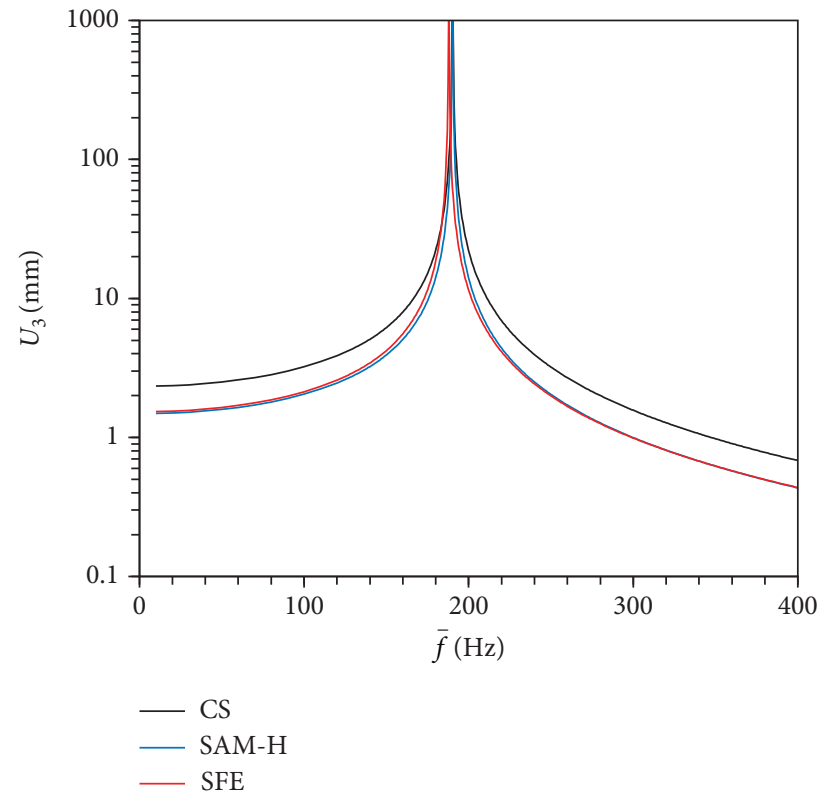

(a)



(b)

FIGURE 2: Displacement amplitude vs. excitation frequency (a); $\sigma_{11}$ and $\sigma_{33}$ stress amplitudes vs. excitation frequency (b). CS, SAM-H, and SFE results.

where $a=3 \mathrm{~m}, s \in[-1,0]$ and $\phi \in[0,2 \pi]$. The curvilinear coordinates $\xi_{1}$ and $\xi_{2}$ are selected as follows:

$$
\begin{aligned}
& \xi_{1}=a(\sinh (s)+\sinh (1)), \\
& \xi_{2}=a \phi \cosh (s) .
\end{aligned}
$$

The principal curvatures have opposite signs: $\kappa_{1}=-\kappa_{2}$. The average thickness-to-radius ratio $\bar{\eta}$ is

$$
\bar{\eta}=\frac{\int_{\omega} \eta \mathrm{d} \omega}{\int_{\omega} \mathrm{d} \omega} \simeq 0.21
$$

and the maximum thickness-to-radius ratio is $\eta_{\max }=0.3$. Consequently, the catenoid is virtually a thick shell. The material is isotropic and its Young's modulus, Poisson's 


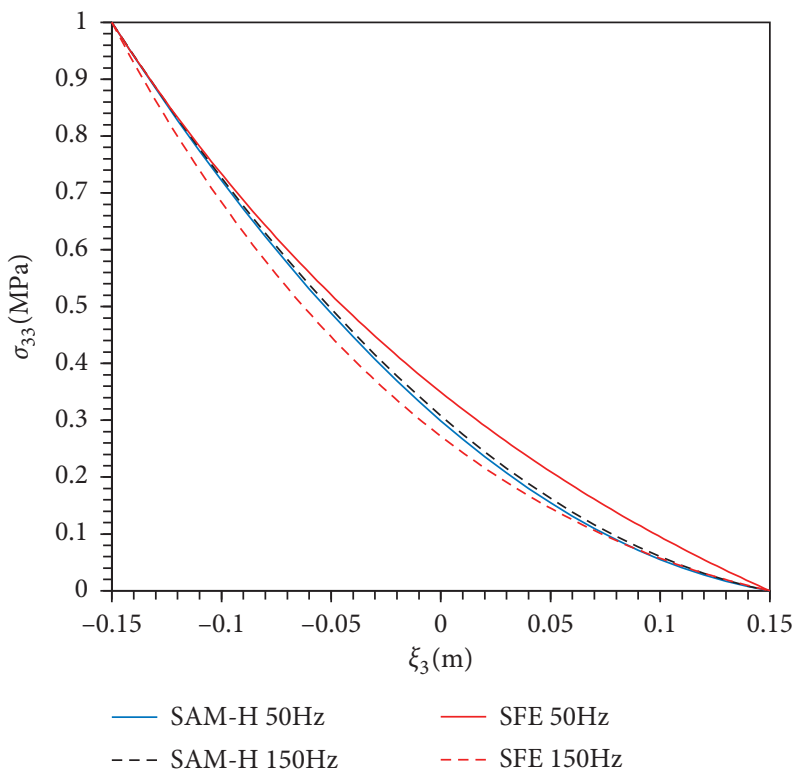

Figure 3: $\sigma_{33}$ amplitude vs. $\xi_{3}$ (SAM-H and SFE results).

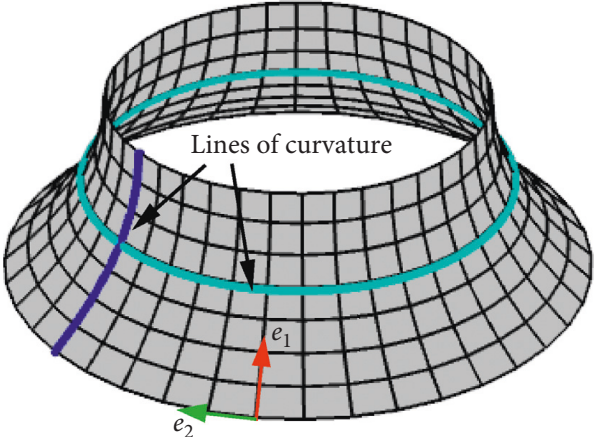

(a)

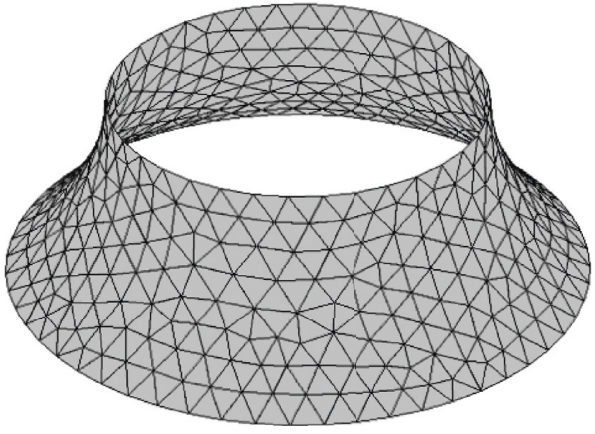

(b)

Figure 4: Catenoid middle surface (a) and example of a 2D meshing (b).

ratio, and density are $E=25 \mathrm{GPa}, \quad \nu=0.2$, and $\rho=$ $2300 \mathrm{~kg} / \mathrm{m}^{3}$, respectively.

For all the shell models (SAM-H, CS, and MITC6), triangular elements with 6 nodes using quadratic polynomials were applied; a quasiuniform element size was selected for the meshing. In Figure 4(b), an example of the meshing of the middle surface is shown. For the solid finite element calculations, tetrahedral quadratic elements were used. All the numerical results of solid finite element computations shown in this section converge.

4.2.1. Eigenfrequencies and Convergence Analysis. The basis of the catenoid is fixed whilst its upper boundary is stress free. This means that for SAM-H and CS models: $U_{1}=U_{2}=$ $U_{3}=0$ and $\phi_{1}=\phi_{2}=0$ at $\xi_{1}=0 ; N_{11}=N_{21}=Q_{1}=0$ and $M_{11}=M_{21}=0$ at $\xi_{1}=a$. No displacement boundary conditions are imposed at the inner and outer faces of the shell. For these shell models, in order to analyze the convergence of results, different meshes were tested; each mesh has a quasiuniform element size and in mesh number $i$, the element size is smaller than in mesh number $i-1$. The first ten eigenfrequencies were calculated, and the approximate error in the calculation of eigenfrequency number $j$ is analyzed:

$$
\delta f_{i}^{j}=\left|\frac{f_{i}^{j}-f_{i-1}^{j}}{f_{i}^{j}}\right| 100 \%,
$$

where $f_{i}^{j}$ is the estimation of eigenfrequency $j$ using mesh $i$. The slowest convergence was observed for the last eigenfrequency $(j=10)$ for all shell computations. In Figure 5, the approximate error $\delta f^{10}$ is plotted against the number of degrees of freedom (DoF) for SAM-H, CS, and MITC6 models. The convergence of both SAM-H and CS calculations is faster than that of MITC6 computations. In what follows, the mesh is selected so as to have values of eigenfrequencies with an approximate error less than $0.01 \%$. In Table 1, the first ten eigenfrequencies calculated by SFE are listed. The eigenfrequencies calculated by SAM-H, CS, and MITC6 models and associated to the eigenmodes related to the SFE eigenfrequencies are also listed in this table. As 


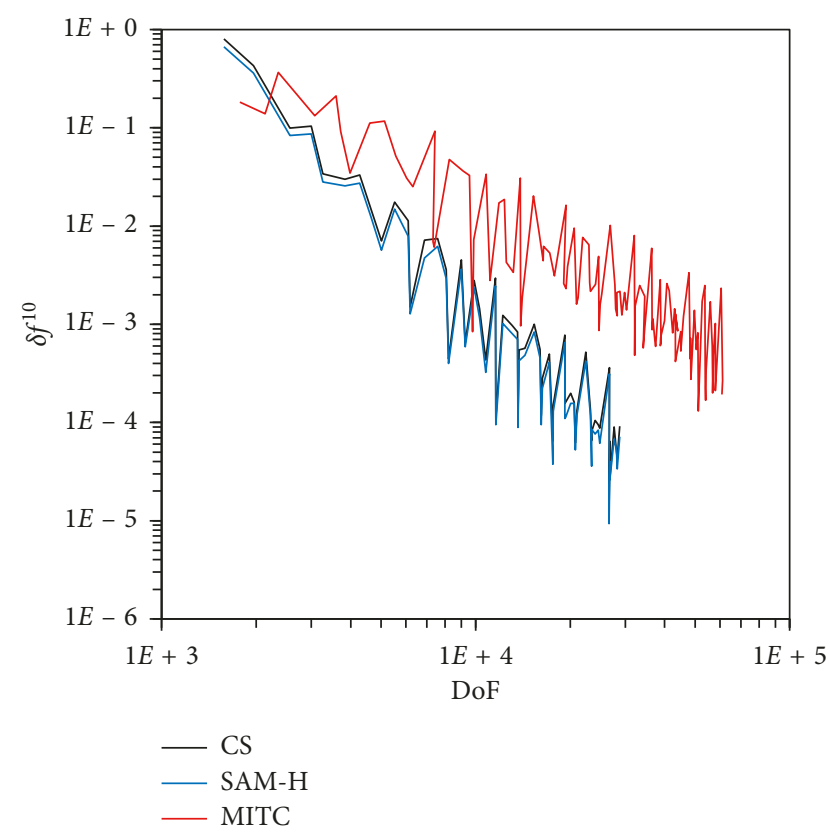

Figure 5: Convergence of SAM-H, CS, and MITC6 models.

TABLE 1: First 10 eigenfrequencies (in $\mathrm{Hz}$ ) computed by SFE, SAM$\mathrm{H}, \mathrm{CS}$, and MITC6.

\begin{tabular}{lcccc}
\hline$j$ & SFE & SAM-H & CS & MITC6 \\
\hline 1,2 & 119.60 & 118.48 & 119.77 & 118.71 \\
3,4 & 122.98 & 121.77 & 122.82 & 122.82 \\
5 & 141.84 & 140.09 & 142.38 & 142.67 \\
6,7 & 154.23 & 152.43 & 154.47 & 151.26 \\
8 & 198.17 & 196.62 & 196.06 & 196.68 \\
9,10 & 198.32 & 196.36 & 196.60 & 191.72 \\
\hline
\end{tabular}

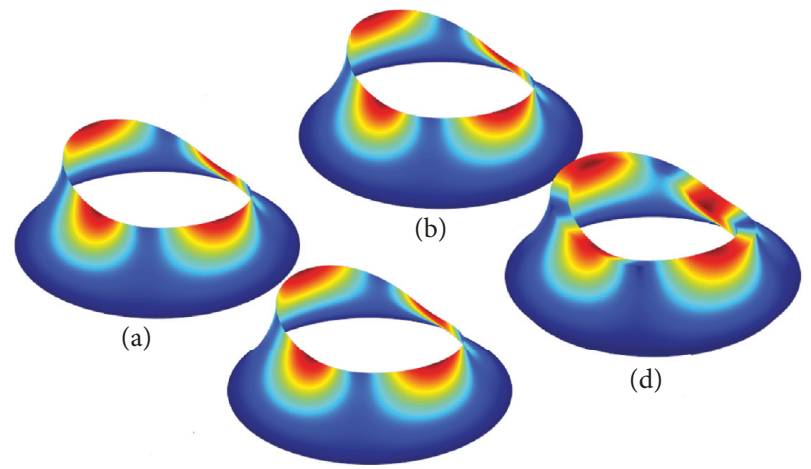

(c)

Figure 6: First eigenmode predicted by (a) SAM-H, (b) CS, (c) MITC6, and (d) SFE models.

compared to SFE results, a relative error less than $4 \%$ is obtained for all shell models considered herein. Since the structure is virtually a thick shell, all models yield good quality results for these eigenfrequencies. In Figure 6, the normalized displacement amplitude of the first mode is shown considering SFE, SAM-H, CS, and MITC6 calculations. Once again, the shell models provide similar eigenmodes to that obtained with SFE.

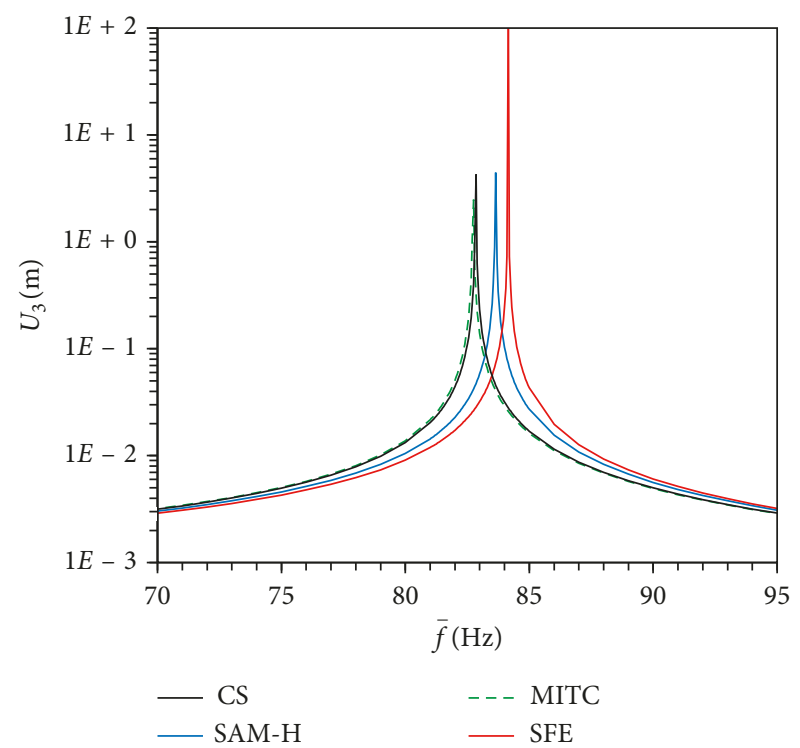

FIGURE 7: Displacement amplitude vs. excitation frequency curve predicted by SFE, SAM-H, CS, and MITC6 models.

4.2.2. Frequency Domain Analysis for the Case of Internal Pressure. The basis of the catenoid remains fixed and the upper edge is stress free. A harmonic pressure $p$ is applied at the inner face of the shell (internal pressure); its amplitude $p_{0}$ is $1 \mathrm{MPa}$, and its frequency $\bar{f}$ is varied so as to perform a frequency domain analysis. The outer face of the shell is stress free. To reduce the computational cost of SFE calculations, the $3 \mathrm{D}$ problem is reduced to a $2 \mathrm{D}$ axisymmetric problem. In Figure 7, the amplitudes of $U_{3}$ at the top of the catenoid calculated by shell models and SFE (at the middle surface) are shown. It can be seen that SAM-H is slightly more accurate than CS and MITC6 for this case. For all the models, a displacement peak is expected at a frequency close to $83 \mathrm{~Hz}$.

For safety reasons, the excitation frequency $\bar{f}$ should be less than $83 \mathrm{~Hz}$. Let us consider the case when $\bar{f}=70 \mathrm{~Hz}$ and analyze the amplitude of stresses along the blue curve parallel to direction 1 shown in Figure 4(a). In Figures 8(a) and $8(\mathrm{~b})$, the amplitudes of the in-plane normal stresses $\sigma_{11}$ and $\sigma_{22}$ at the internal face of the shell $\left(\xi_{3}=-(h / 2)\right)$ are plotted against the $\xi_{1}$ coordinate, respectively. Globally, the shape and maximum values of the SFE curves in Figures 8(a) and 8 (b) are approximated better by SAM-H calculations. It is worth mentioning that $\sigma_{22}$ exhibits the highest value among the other stress components in the curvilinear coordinate system. As compared to SFE, the relative errors in the maximum value of $\sigma_{22}$ predicted by SAM-H, MITC6 and CS models are $2.8 \%,-9.7 \%$, and $-10.5 \%$, respectively. SAM$\mathrm{H}$ in-plane normal stresses are then more accurate than those of CS and MITC6 techniques.

In Figure 9(a) the amplitude of the shear stress $\sigma_{13}$ at the middle surface is plotted against the curvilinear coordinate $\xi_{1}$. The evolution of the SFE $\sigma_{13}$ stress along the line of curvature is approximated more accurately by SAM-H than MITC6 and CS models. The maximum value of the SFE shear stress is better approximated by SAM-H and MITC6 


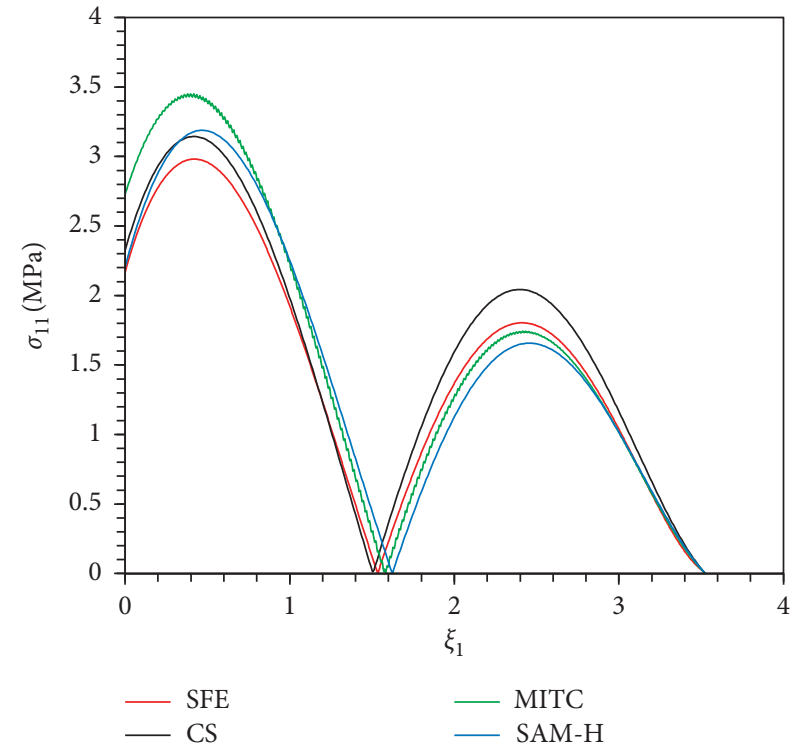

(a)



(b)

FIGURE 8: $\sigma_{11}$ vs. $\xi_{1}$ (a) and $\sigma_{22}$ vs. $\xi_{1}$ (b) curves at the external face of the shell obtained by SFE, CS, MITC6, and SAM-H models.



(a)



(b)

Figure 9: $\sigma_{13}$ vs. $\xi_{1}$ (a) and $\sigma_{33}$ vs. $\xi_{1}$ (b) curves at the middle surface of the shell obtained by SFE, CS, MITC6, and SAM-H models.

models than by the CS model. Let us now analyze the accuracy of SAM-H in the calculation of the out-of-plane normal stress $\sigma_{33}$. It is worth recalling that CS and MITC6 techniques do not calculate this stress. At the inner and outer faces of the shell, since SAM-H verifies the 3D boundary conditions, SAM-H yields the amplitudes $\sigma_{33}=1 \mathrm{MPa}$ and $\sigma_{33}=0 \mathrm{MPa}$, respectively. At the middle surface, the amplitudes of the stresses $\sigma_{33}$ calculated by SFE and SAM-H are plotted against $\xi_{1}$ in Figure 9(b). As in the case of the pressurized hollow sphere in Section 4.1, SAM-H yields good quality predictions of $\sigma_{33}$.

\section{Conclusions}

To conclude, a mixed stress/displacement approach model for dynamic problems of moderately thick shells was developed. It is an extension of the static SAM-H model developed by Domínguez Alvarado and Díaz in [1]; for this reason, the name SAM-H was preserved for the dynamic model. The dynamic model equations were obtained by introducing the Hellinger-Reissner functional into a Lagrangian and applying the Euler-Lagrange equation. The main goal of SAM-H is to determine accurately the $3 \mathrm{D}$ 
stresses: their approximation is inspired by the static version of SAM-H. The 3D displacement approximation is required to obtain the generalized motion equations: an approximation equivalent to that in first-order displacement approach models was chosen. The stress approximation verifies the 3D stress boundary conditions at the faces of the shell and the 3D motion equations. The constitutive equations take into account Poisson's effect of out-of-plane normal stresses on in-plane strains; this is an important feature which is ignored in most shell models. Two structures were analyzed to test the accuracy of SAM-H: a hollow sphere and a catenoid. An analytical resolution was proposed for the study of the first structure whilst a finite element method was adopted to solve the model equations for the analysis of the second one. For both structures, eigenfrequencies were determined; after this, a harmonic internal pressure was applied and a frequency domain analysis was performed. SAM-H results were compared to those of MITC6 surface elements, the classic shell (CS) model, and solid finite elements (SFE). Both shell models and MITC6 elements yield accurate eigenfrequencies and eigenmodes even for a thick catenoid; their values are virtually the same and similar to those of SFE. Nevertheless, in a frequency domain analysis, the amplitudes of displacements and stresses of CS and MITC6 are not as accurate as those of SAM-H model which approximates SFE results with great precision.

The numerical resolution of SAM-H equations does not imply a higher computational cost than that of the CS model because the same number of generalized displacements are considered. Besides, we have proved that the finite element calculations for the resolution of SAM-H equations converge faster than MITC6 calculations. The polynomial stress approximation in SAM-H may seem complex, but since the stress calculation should be performed in a postprocessing stage, it does not represent a computational drawback. For these reasons, SAM-H can be implemented in commercial finite element software for the dynamic analysis of homogeneous, moderately thick shells: calculation of eigenfrequencies and eigenmodes, frequency domain analysis, transient response, etc.

\section{Appendix}

\section{A. Coefficients of the Polynomial Approximation of Stresses}

In this section, the stress coefficients in the polynomial approximations appearing in equation (13)-(15) are detailed. The following expressions are obtained:

(i) For $\sigma_{\alpha \beta}^{n}(0 \leq n \leq 1)$ :

$$
\left\{\begin{array}{l}
\sigma_{\alpha \beta}^{0}=\frac{1}{h}\left[(\underline{\underline{\mathbf{N}}}-\underline{\underline{\mathbf{M}}} \cdot \underline{\underline{\mathbf{\kappa}}}) \cdot \mathbf{e}_{\boldsymbol{\beta}}\right] \cdot \mathbf{e}_{\boldsymbol{\alpha}}, \\
\sigma_{\alpha \beta}^{1}=\left[\left(\frac{12}{h^{2}} \underline{\underline{\mathbf{M}}}-\underline{\underline{\mathbf{N}} \cdot \underline{\underline{\mathbf{k}}}}\right) \cdot \mathbf{e}_{\boldsymbol{\beta}}\right] \cdot \mathbf{e}_{\boldsymbol{\alpha}} .
\end{array}\right.
$$

$$
\left\{\begin{aligned}
\sigma_{\alpha 3}^{0}= & {\left[\frac{1}{h} \mathbf{Q}-\frac{h}{12} \underline{\mathbf{k}}^{\prime} \cdot\left(\underline{\boldsymbol{\tau}}^{+}+\underline{\boldsymbol{\tau}}^{-}\right)\right] \cdot \mathbf{e}_{\boldsymbol{\alpha}}, } \\
\sigma_{\alpha 3}^{1}= & \left(\underline{\boldsymbol{\tau}}^{+}+\underline{\boldsymbol{\tau}^{-}}\right) \cdot \mathbf{e}_{\boldsymbol{\alpha}}+\frac{1}{5} \sigma_{\alpha 3}^{3}, \\
\sigma_{\alpha 3}^{2}= & {\left[\frac{1}{h} \mathbf{Q}-\frac{1}{2}\left(\underline{\boldsymbol{\tau}}^{+}+\underline{\boldsymbol{\tau}}^{-}\right)-\frac{h}{12} \underline{\underline{\mathbf{\kappa}}} \cdot\left(\underline{\boldsymbol{\tau}}^{+}+\underline{\boldsymbol{\tau}}^{-}\right)\right] \cdot \mathbf{e}_{\boldsymbol{\alpha}} } \\
\sigma_{\alpha 3}^{3}= & {\left[2 \underline{\mathbf{d i v}}\left(\underline{\underline{\mathbf{M}}} \cdot \underline{\underline{\kappa^{\prime}}}\right)-2\left(\kappa_{1}+\kappa_{2}\right) \underline{\mathbf{d i v}} \underline{\underline{\mathbf{M}}}\right] \cdot \mathbf{e}_{\boldsymbol{\alpha}} } \\
& +\left(\kappa_{1}+\kappa_{2}\right)\left[h\left(\underline{\boldsymbol{\tau}}^{+}+\underline{\boldsymbol{\tau}}^{-}\right)-2 \underline{\mathbf{Q}}\right] \cdot \mathbf{e}_{\boldsymbol{\alpha}} \\
& -\left(4 \underline{\underline{\kappa}}+3 \underline{\underline{\kappa}}^{\prime}\right) \cdot\left(\underline{\mathbf{Q}}-\frac{h}{2}\left(\underline{\boldsymbol{\tau}}^{+}+\underline{\boldsymbol{\tau}^{-}}\right)\right) \cdot \mathbf{e}_{\boldsymbol{\alpha}} .
\end{aligned}\right.
$$

(iii) For $\sigma_{33}^{n}(0 \leq n \leq 4)$ :

$$
\begin{aligned}
& \left\{\begin{array}{l}
\sigma_{33}^{0}=\frac{\sigma^{+}-\sigma^{-}}{2}+\sigma_{33}^{2}+\frac{1}{15} \sigma_{33}^{4}, \\
\sigma_{33}^{1}=\sigma^{+}+\sigma^{-}+\frac{1}{5} \sigma_{33}^{3},
\end{array}\right. \\
& \sigma_{33}^{2}=\frac{h}{30} \operatorname{div}[\underline{\operatorname{div}}(\underline{\underline{\mathbf{M}}} \cdot \underline{\underline{\mathbf{k}}})-2 \underline{\underline{\mathbf{k}}} \cdot \underline{Q}]-\frac{1}{h} \underline{\underline{\mathbf{M}}}: \underline{\underline{\mathbf{k}}}-\frac{1}{15} \sigma_{33}^{4} \\
& +\frac{h}{12} \operatorname{div}\left[\underline{\tau}^{+}-\underline{\tau}^{-}+\frac{h}{10}(4 \underline{\underline{\boldsymbol{\kappa}}}+5 \underline{\underline{\underline{\kappa}}}) \cdot\left(\underline{\tau}^{+}-\underline{\tau}^{-}\right)\right] \\
& +\frac{h}{12}\left(\kappa_{1}+\kappa_{2}\right)\left(\sigma_{33}^{1}-\frac{2}{5} \sigma_{33}^{3}+\frac{4}{5} \sigma_{33}^{4}-\operatorname{div} \underline{Q}\right) \\
& -\frac{h}{30} \operatorname{div}\left[\left(\underline{\underline{\mathbf{\kappa}}}+\underline{\underline{\mathbf{k}^{\prime}}}\right) \cdot \underline{\mathbf{d i v}} \underline{\underline{\mathbf{M}}}\right]+\frac{h}{30} \operatorname{div}[(\underline{\underline{\mathbf{\kappa}}}+\underline{\underline{\mathbf{k}}}) \cdot \underline{Q}] \\
& -\frac{h^{2}}{60} \operatorname{div}\left[\left(\underline{\underline{\mathbf{\kappa}}}+\underline{\underline{\boldsymbol{k}^{\prime}}}\right) \cdot\left(\underline{\tau}^{+}-\underline{\tau}^{-}\right)\right], \\
& \sigma_{33}^{3}=-\operatorname{div} \underline{Q}+\frac{h}{4} \operatorname{div}\left[2\left(\underline{\tau}^{+}-\underline{\tau}^{-}\right)-h \underline{\underline{\mathbf{\kappa}}} \cdot\left(\underline{\tau}^{+}-\underline{\tau}^{-}\right)\right], \\
& \sigma_{33}^{4}=-\frac{3 h}{14} \operatorname{div}\left[\underline{\operatorname{div}}\left(\underline{\underline{\mathbf{M}}} \cdot \underline{\underline{\boldsymbol{\kappa}^{\prime}}}\right)\right]+\frac{3 h}{7} \operatorname{div}\left[\left(2 \underline{\underline{\mathbf{\kappa}}}+\underline{\underline{\boldsymbol{\kappa}^{\prime}}}\right) \cdot \underline{Q}\right] \\
& -\frac{3 h^{2}}{14} \operatorname{div}\left[\left(2 \underline{\underline{\underline{\kappa}}}+\underline{\underline{\underline{k}}}^{\prime}\right) \cdot\left(\underline{\tau}^{+}-\underline{\tau}^{-}\right)\right] \\
& +\frac{3 h}{14} \operatorname{div}\left[\left(\underline{\underline{\mathbf{\kappa}}}+\underline{\underline{\boldsymbol{\kappa}^{\prime}}}\right) \cdot \underline{\mathbf{d i v}} \underline{\underline{\mathbf{M}}}\right]-\frac{3 h}{14} \operatorname{div}[(\underline{\underline{\mathbf{\kappa}}}+\underline{\underline{\mathbf{k}}}) \cdot \underline{Q}] \\
& +\frac{3 h^{2}}{28} \operatorname{div}\left[(\underline{\underline{\mathbf{k}}}+\underline{\underline{\underline{\mathbf{k}}}}) \cdot\left(\underline{\tau}^{+}-\underline{\tau}^{-}\right)\right] \text {. }
\end{aligned}
$$

(ii) For $\sigma_{\alpha 3}^{n}(0 \leq n \leq 3)$ : 


\section{B. Simplification of the Elastic Energy}

In this section, a method to express the elastic energy as an algebraic function of the generalized forces is proposed. This energy is defined by

$$
\int_{\Omega} w_{e} \mathrm{~d} \Omega
$$

where the elastic energy density $w_{e}\left(\boldsymbol{\sigma}^{*}\right)=(1 / 2) \boldsymbol{\sigma}^{*}: \mathbf{S}: \boldsymbol{\sigma}^{*}$ must be expressed in terms of the generalized forces. The stress coefficients appearing in equations (A.1)-(A.3) involve the generalized forces but also their divergences. These divergences complicate the calculation of the elastic energy; some simplifications must be adopted. The following stress coefficients are simplified as follows:

$$
\begin{aligned}
& \sigma_{13}^{3} \simeq-\left(3 \kappa_{1}+2 \kappa_{2}\right) \cdot\left(Q_{1}-\frac{h}{2}\left(\tau_{1}^{+}-\tau_{1}^{-}\right)\right), \\
& \sigma_{23}^{3} \simeq-\left(3 \kappa_{2}+2 \kappa_{1}\right) \cdot\left(Q_{2}-\frac{h}{2}\left(\tau_{2}^{+}-\tau_{2}^{-}\right)\right) \text {, } \\
& \sigma_{33}^{2} \simeq \frac{h}{60}\left(\kappa_{1}+\kappa_{2}\right)\left(\sigma^{+}+\sigma^{-}\right)+\frac{h^{2}}{10}\left(\kappa_{1}+\kappa_{2}\right) f_{3}-\frac{1}{h} \underline{\underline{\mathbf{M}}}: \underline{\underline{\mathbf{\kappa}}} \\
& +\frac{h}{12}\left[\left(\kappa_{1}+\kappa_{2}\right)\left(2 \sigma_{33}^{1}+\frac{3}{5} \sigma_{33}^{3}\right)-\frac{4}{5 h} \sigma_{33}^{4}\right], \\
& \sigma_{33}^{3} \simeq-\underline{\underline{\mathbf{N}}}: \underline{\underline{\boldsymbol{\kappa}}}+h f_{3}+h\left(\kappa_{1}+\kappa_{2}\right) \frac{\sigma^{+}-\sigma^{-}}{2}+\sigma^{+}+\sigma^{-} \text {, } \\
& \sigma_{33}^{4} \simeq-\frac{15 h}{28}\left(\kappa_{1}+\kappa_{2}\right)\left(\sigma^{+}+\sigma^{-}+f_{3} h\right) .
\end{aligned}
$$

It is worth mentioning that this simplification is used exclusively for the calculation of the elastic energy. In a stress analysis stage, which assumes that the generalized forces were previously obtained, equations (A.1)-(A.3) are used without any simplification.

\section{Generalized Stiffnesses}

In what follows, the generalized stiffnesses are expressed in terms of inverses of the components $S_{i j k l}$ of the 3D compliance tensor S. It is worth adopting the following notations:

$$
\begin{aligned}
& S_{\alpha \beta}^{c}=2 \times S_{\alpha \beta 33}, \\
& S_{\alpha \beta}^{Q}=4 \times S_{\alpha 3 \beta 3}, \\
& S^{\sigma}=S_{3333} .
\end{aligned}
$$

The coefficients of the stiffness matrices and vectors shown in equation (25) are

(i) Matrix $\mathbf{K}$ is defined by

$$
K=\left(\begin{array}{ll}
A & B \\
C & D
\end{array}\right) \text {, }
$$

where $\left\{\begin{array}{l}\mathbf{A}=\mathbf{G}^{1} \check{\mathbf{A}} \mathbf{G}^{2}+\left(\kappa_{2}-\kappa_{1}\right) \mathbf{G}^{3} \check{\mathbf{C}}^{2}, \\ \mathbf{B}=\mathbf{G}^{1} \check{\mathbf{B}} \mathbf{G}^{2}+\left(\kappa_{2}-\kappa_{1}\right) \mathbf{G}^{3} \check{\mathbf{D}}^{2}, \\ \mathbf{C}=\mathbf{G}^{1} \check{\mathbf{C}} \mathbf{G}^{2}+\frac{h^{2}}{12}\left(\kappa_{2}-\kappa_{1}\right) \mathbf{G}^{3} \check{\mathbf{A}}^{2}, \\ \mathbf{D}=\mathbf{G}^{1} \check{\mathbf{D}} \mathbf{G}^{2}+\frac{h^{2}}{12}\left(\kappa_{2}-\kappa_{1}\right) \mathbf{G}^{3} \check{\mathbf{B}}^{2} .\end{array}\right.$

In the equation above, matrices $\mathbf{G}^{1}, \mathbf{G}^{2}$, and $\mathbf{G}^{3}$ stand for

$$
\begin{aligned}
\mathbf{G}^{1} & =\left(\begin{array}{lll}
1 & 0 & 0 \\
0 & 1 & 0 \\
0 & 0 & 1 \\
0 & 0 & 1
\end{array}\right), \\
\mathbf{G}^{2} & =\left(\begin{array}{llll}
1 & 0 & 0 & 0 \\
0 & 1 & 0 & 0 \\
0 & 0 & 1 & 1
\end{array}\right), \\
\mathbf{G}^{3} & =\left(\begin{array}{lll}
0 & 0 & 0 \\
0 & 0 & 0 \\
0 & 0 & 0 \\
0 & 0 & 1
\end{array}\right) .
\end{aligned}
$$

Matrices $\check{\mathbf{A}}, \check{\mathbf{B}}, \check{\mathbf{C}}$, and $\check{\mathbf{D}}$ appearing in equation (C.3) are defined by

$$
\left\{\begin{array}{l}
\check{\mathbf{A}}=\left(\check{\mathbf{C}}^{\mathbf{A}}\right)^{-1}, \\
\check{\mathbf{B}}=-\check{\mathbf{A}}\left(\check{\mathbf{C}}^{\mathbf{B} 1}+\check{\mathbf{C}}^{\mathbf{B} 2}\right) \check{\mathbf{D}}, \\
\check{\mathbf{C}}=-\check{\mathbf{D}}\left(\check{\mathbf{C}}^{\mathrm{C} 1}+\check{\mathbf{C}}^{\mathbf{C} 2}\right) \check{\mathbf{A}}, \\
\check{\mathbf{D}}=\frac{h^{2}}{12} \check{\mathbf{A}},
\end{array}\right.
$$

where 


$$
\begin{aligned}
& \check{\mathbf{C}}^{\mathbf{A}}=\frac{1}{h}\left(\begin{array}{ccc}
S_{1111} & S_{1122} & 2 S_{1112} \\
S_{1122} & S_{2222} & 2 S_{2212} \\
2 S_{1112} & 2 S_{2212} & 4 S_{1212}
\end{array}\right), \\
& \check{\mathbf{C}}^{\mathbf{B} 1}=\check{\mathbf{C}}^{\mathrm{C} 1}=\frac{\kappa_{1}-\kappa_{2}}{h}\left(\begin{array}{ccc}
S_{1111} & 0 & 0 \\
0 & -S_{2222} & -2 S_{2212} \\
S_{1112} & -S_{2212} & -2 S_{1212}
\end{array}\right) \text {, } \\
& \check{\mathbf{C}}^{\mathbf{B} 2}=-\frac{1}{10 h}\left(\begin{array}{ccc}
6 S_{11}^{C} \kappa_{1} & S_{22}^{C} \kappa_{1}+5 S_{11}^{C} \kappa_{2} & 2 S_{12}^{C} \kappa_{1} \\
S_{11}^{C} \kappa_{2}+5 S_{22}^{C} \kappa_{1} & 6 S_{22}^{C} \kappa_{2} & 2 S_{12}^{C} \kappa_{2} \\
10 S_{12}^{C} \kappa_{1} & 10 S_{12}^{C} \kappa_{2} & 0
\end{array}\right), \\
& \check{\mathbf{C}}^{\mathrm{C} 2}=-\frac{1}{10 h}\left(\begin{array}{ccc}
6 S_{11}^{C} \kappa_{1} & 5 S_{22}^{C} \kappa_{1}+S_{11}^{C} \kappa_{2} & 10 S_{12}^{C} \kappa_{1} \\
5 S_{11}^{C} \kappa_{2}+S_{22}^{C} \kappa_{1} & 6 S_{22}^{C} \kappa_{2} & 10 S_{12}^{C} \kappa_{2} \\
2 S_{12}^{C} \kappa_{1} & 2 S_{12}^{C} \kappa_{2} & 0
\end{array}\right) .
\end{aligned}
$$

(ii) Vectors $\mathbf{k}^{+}, \mathbf{k}^{-}$, and $\mathbf{k}^{3}$ are defined by

$$
\left\{\begin{array}{l}
\mathbf{k}^{+}=\left(\begin{array}{c}
\mathbf{G}^{1} \check{\mathbf{k}}_{N}^{+}+\left(\kappa_{2}-\kappa_{1}\right) \mathbf{G}^{3} \check{\mathbf{k}}_{M}^{+} \\
\mathbf{G}^{1} \check{\mathbf{k}}_{M}^{+}+\frac{h^{2}}{12}\left(\kappa_{2}-\kappa_{1}\right) \mathbf{G}^{3} \check{\mathbf{k}}_{N}^{+}
\end{array}\right), \\
\mathbf{k}^{-}=\left(\begin{array}{c}
\mathbf{G}^{1} \check{\mathbf{k}}_{N}^{-}+\left(\kappa_{2}-\kappa_{1}\right) \mathbf{G}^{3} \check{\mathbf{k}}_{M}^{-} \\
\mathbf{G}^{1} \check{\mathbf{k}}_{M}^{-}+\frac{h^{2}}{12}\left(\kappa_{2}-\kappa_{1}\right) \mathbf{G}^{3} \check{\mathbf{k}}_{N}^{-}
\end{array}\right), \\
\mathbf{k}^{3}=\left(\begin{array}{c}
\mathbf{G}^{1} \check{\mathbf{k}}_{N}^{3}+\left(\kappa_{2}-\kappa_{1}\right) \mathbf{G}^{3} \check{\mathbf{k}}_{M}^{3} \\
\mathbf{G}^{1} \check{\mathbf{k}}_{M}^{3}+\frac{h^{2}}{12}\left(\kappa_{2}-\kappa_{1}\right) \mathbf{G}^{3} \check{\mathbf{k}}_{N}^{3}
\end{array}\right),
\end{array}\right.
$$

where

$$
\begin{aligned}
& \left(\check{\mathbf{k}}_{N}^{+}=\check{\mathbf{B}}_{\chi}^{+}+\check{\mathbf{A}} \check{\mathbf{c}}_{\varepsilon}^{+},\right. \\
& \check{\mathbf{k}}_{N}^{-}=\check{\mathbf{B}} \check{\mathbf{c}}_{\chi}^{-}+\check{\mathbf{A}} \check{\mathbf{c}}_{\varepsilon}^{-} \text {, } \\
& \check{\mathbf{k}}_{N}^{3}=\check{\mathbf{B}} \check{\mathbf{c}}_{\chi}^{3}+\check{\mathbf{A}} \check{\mathbf{c}}_{\varepsilon}^{3}, \\
& \check{\mathbf{k}}_{M}^{+}=\check{\mathbf{C}} \check{\mathbf{c}}_{\varepsilon}^{+}+\check{\mathbf{D}} \check{\mathbf{c}}_{\chi}^{+}, \\
& \check{\mathbf{k}}_{M}^{-}=\check{\mathbf{C}} \check{\mathbf{c}}_{\varepsilon}^{-}+\check{\mathrm{D}} \check{\mathbf{c}}_{\chi}^{-}, \\
& \check{\mathbf{k}}_{M}^{3}=\check{\mathbf{C}}_{\boldsymbol{\varepsilon}}^{3}+\check{\mathbf{D}} \check{\mathbf{c}}_{\chi}^{3} \\
& \check{\mathbf{c}}_{\varepsilon}^{+}=3 h \frac{S^{\sigma}}{140} \check{\mathbf{c}}^{\mathbf{a}}-\frac{1}{60} \check{\mathbf{C}}^{\mathbf{s}}\left(15 \check{\mathbf{c}}^{\mathbf{b}}+8 h \check{\mathbf{c}}^{\mathbf{c}}+5 h \check{\mathbf{c}}^{\mathbf{d}}\right), \\
& \check{\mathbf{c}}_{\boldsymbol{\varepsilon}}^{-}=3 h \frac{S^{\sigma}}{140} \check{\mathbf{c}}^{\mathbf{a}}-\frac{1}{60} \check{\mathbf{C}}^{\mathbf{S}}\left(-15 \check{\mathbf{c}}^{\mathbf{b}}+8 h \check{\mathbf{c}}^{\mathbf{c}}+5 h \check{\mathbf{c}}^{\mathbf{d}}\right), \\
& \check{\mathbf{c}}_{\varepsilon}^{3}=\frac{h^{2} S^{\sigma}}{210} \check{\mathbf{c}}^{\mathbf{a}}-\frac{1}{120} h^{2} \check{\mathbf{C}}^{\mathbf{S}}\left(6 \check{\mathbf{c}}^{\mathbf{c}}+5 \check{\mathbf{c}}^{\mathbf{d}}\right), \\
& \check{\mathbf{c}}_{\chi}^{+}=\frac{S^{\sigma}}{2} \check{\mathbf{c}}^{\mathbf{a}}-\frac{1}{60 h} \check{\mathbf{C}}^{\mathbf{S}}\left(36 \check{\mathbf{c}}^{\mathbf{b}}+18 h \check{\mathbf{c}}^{\mathbf{c}}+3 h \check{\mathbf{c}}^{\mathbf{d}}\right), \\
& \check{\mathbf{c}}_{x}^{-}=-\frac{S^{\sigma}}{2} \check{\mathbf{c}}^{\mathbf{a}}-\frac{1}{60 h} \check{\mathbf{C}}^{\mathbf{S}}\left(36 \check{\mathbf{c}}^{\mathbf{b}}-18 h \check{\mathbf{c}}^{\mathbf{c}}-3 h \check{\mathbf{c}}^{\mathbf{d}}\right) \text {, } \\
& \check{\mathbf{c}}_{\chi}^{3}=-\frac{1}{10} \check{\mathbf{C}} \check{\mathrm{c}}^{\mathrm{b}} \text {. }
\end{aligned}
$$

In the definitions above, matrix $\check{\mathbf{C}}^{\mathbf{S}}$ and vectors $\check{\mathbf{c}}^{\mathbf{a}}, \check{\mathbf{c}}^{\mathbf{b}}$, and $\check{c}^{\mathfrak{c}}$ stand for

$$
\left\{\begin{aligned}
& \check{\mathbf{C}}^{\mathbf{S}}=\left(\begin{array}{ccc}
S_{11}^{C} & 0 & 0 \\
0 & S_{22}^{C} & 0 \\
0 & 0 & S_{12}^{C}
\end{array}\right), \\
& \check{\mathbf{c}}^{\mathbf{a}}=\left(\begin{array}{c}
\kappa_{1} \\
\kappa_{2} \\
0
\end{array}\right), \\
& \check{\mathbf{c}}^{\mathbf{b}}=\left(\begin{array}{c}
1 \\
1 \\
2
\end{array}\right) \\
& \check{\mathbf{c}}^{\mathbf{c}}=\left(\begin{array}{c}
\kappa_{1} \\
\kappa_{2} \\
\kappa_{1}+\kappa_{2}
\end{array}\right), \\
& \check{\mathbf{c}}^{\mathbf{d}}=\left(\begin{array}{c}
\kappa_{2} \\
\kappa_{1} \\
\kappa_{1}+\kappa_{2}
\end{array}\right) .
\end{aligned}\right.
$$

(iii) Matrices $\mathbf{L}, \mathbf{L}^{+}$, and $\mathbf{L}^{-}$are defined by 


$$
\left\{\begin{array}{l}
\mathbf{L}=\frac{6}{5 h}\left(\begin{array}{cc}
S_{11}^{Q} & S_{12}^{Q} \\
S_{21}^{Q} & S_{22}^{Q}
\end{array}\right)^{-1}, \\
\mathbf{L}^{+}=\frac{1}{60} \mathbf{L} \cdot\left(\begin{array}{cc}
S_{11}^{Q}\left(6+5 h \kappa_{2}\right) & S_{12}^{Q}\left(6+6 h \kappa_{1}-h \kappa_{2}\right) \\
S_{21}^{Q}\left(6-h \kappa_{1}+6 h \kappa_{2}\right) & S_{22}^{Q}\left(6+5 h \kappa_{1}\right)
\end{array}\right) \\
\mathbf{L}^{-}=-\frac{1}{60} \mathbf{L} \cdot\left(\begin{array}{cc}
S_{11}^{Q}\left(6-5 h \kappa_{2}\right) & S_{12}^{Q}\left(6-6 h \kappa_{1}+h \kappa_{2}\right) \\
S_{21}^{Q}\left(6+h \kappa_{1}-6 h \kappa_{2}\right) & S_{22}^{Q}\left(6-5 h \kappa_{1}\right)
\end{array}\right)
\end{array}\right.
$$

It is worth mentioning that some of the components of the vectors defined in equation (C.8) can still be simplified because they involve $\left(\kappa_{1} h\right)^{2},\left(\kappa_{2} h\right)^{2}$ or $\kappa_{1} \kappa_{2} h^{2}$ terms that are neglected in the model.

\section{Data Availability}

The data used to support the findings of this study are included within the article.

\section{Conflicts of Interest}

The authors declare that they have no conflicts of interest.

\section{References}

[1] A. F. Domínguez Alvarado and A. D. Díaz, "A stress approach model of moderately thick, homogeneous shells," Mathematical Problems in Engineering, vol. 2018, Article ID 6141683, 16 pages, 2018.

[2] A. E. H. Love, "The small free vibrations and deformation of a thin elastic shell," Philosophical Transactions of the Royal Society A: Mathematical, Physical and Engineering Sciences, vol. 179, pp. 491-546, 1888 .

[3] E. Ventsel and T. Krauthammer, Thin Plates and Shells: Theory, Analysis, and Applications, Marcel Dekker, New York, NY, USA, 2001.

[4] P. M. Naghdi, "The theory of shells and plates," in Linear Theories of Elasticity and Thermoelasticity, pp. 425-640, Springer, Berlin, Germany, 1973.

[5] J. N. Reddy, Theory and Analysis of Elastic Plates and Shells, CRC Press, Boca Raton, FL, USA, 2006.

[6] Y.-G. Fang, J.-H. Pan, and W.-X. Chen, "Theory of thickwalled shells and its application in cylindrical shell," Applied Mathematics and Mechanics, vol. 13, no. 11, pp. 1055-1065, 1992.

[7] G. Z. Voyiadjis and P. Woelke, "A refined theory for thick spherical shells," International Journal of Solids and Structures, vol. 41, no. 14, pp. 3747-3769, 2004.

[8] G. Z. Voyiadjis and G. Shi, "A refined two-dimensional theory for thick cylindrical shells," International Journal of Solids and Structures, vol. 27, no. 3, pp. 261-282, 1991.

[9] E. Carrera, "Theories and finite elements for multilayered, anisotropic, composite plates and shells," Archives of Computational Methods in Engineering, vol. 9, no. 2, pp. 87-140, 2002.
[10] J. L. Sanders Jr., An Improved First-Approximation Theory for Thin Shells, NASA TR-R24. US Government Printing Office, Washington, DC, USA, 1959.

[11] F. Tornabene and E. Viola, "Static analysis of functionally graded doubly-curved shells and panels of revolution," Meccanica, vol. 48, no. 4, pp. 901-930, 2013.

[12] E. Viola, F. Tornabene, and N. Fantuzzi, "Static analysis of completely doubly-curved laminated shells and panels using general higher-order shear deformation theories," Composite Structures, vol. 101, pp. 59-93, 2013.

[13] E. Trefftz, "Ableitung der Schalenbiegungsgleichungen mit dem Castiglianoschen Prinzip," ZAMM-Journal of Applied Mathematics and Mechanics/Zeitschrift für Angewandte Mathematik und Mechanik, vol. 15, no. 1-2, pp. 101-108, 1935.

[14] J. L. Synge and W. Z. Chien, "The intrinsic theory of elastic shells and plates," Von Kármán Anniversary, vol. 2, pp. 103-120, 1941.

[15] W.-Z. Chien, "The intrinsic theory of thin shells and plates: part I.-general theory," Quarterly of Applied Mathematics, vol. 1, no. 4, pp. 297-327, 1944.

[16] E. Reissner, "On a variational theorem in elasticity," Journal of Mathematics and Physics, vol. 29, no. 1-4, pp. 90-95, 1950.

[17] B. Liu, Y. F. Xing, M. S. Qatu, and A. J. M. Ferreira, "Exact characteristic equations for free vibrations of thin orthotropic circular cylindrical shells," Composite Structures, vol. 94, no. 2, pp. 484-493, 2012.

[18] F. Tornabene, A. Liverani, and G. Caligiana, "General anisotropic doubly-curved shell theory: a differential quadrature solution for free vibrations of shells and panels of revolution with a free-form meridian," Journal of Sound and Vibration, vol. 331, no. 22, pp. 4848-4869, 2012.

[19] F. Tornabene, N. Fantuzzi, and M. Bacciocchi, "Free vibrations of free-form doubly-curved shells made of functionally graded materials using higher-order equivalent single layer theories," Composites Part B: Engineering, vol. 67, pp. 490-509, 2014.

[20] F. Tornabene, "General higher-order layer-wise theory for free vibrations of doubly-curved laminated composite shells and panels," Mechanics of Advanced Materials and Structures, vol. 23, no. 9, pp. 1046-1067, 2016.

[21] Q. Wang, D. Shi, Q. Liang, and F. Pang, "Free vibration of fourparameter functionally graded moderately thick doubly-curved panels and shells of revolution with general boundary conditions," Applied Mathematical Modelling, vol. 42, pp. 705-734, 2017.

[22] A. W. Leissa, "Vibration of shells," Technical Report, NASA, Washington, DC, USA, 1973.

[23] W. Flügge, Stresses in Shells, Springler-Verlag, Berlin, Germany, 1966.

[24] R. Poultangari and M. Nikkhah-Bahrami, "Free and forced vibration analysis of stepped circular cylindrical shells with several intermediate supports using an extended wave method; a generalized approach," Latin American Journal of Solids and Structures, vol. 13, no. 11, pp. 2027-2058, 2016.

[25] M. L. Bucalem and K.-J. Bathe, "Higher-order mitc general shell elements," International Journal for Numerical Methods in Engineering, vol. 36, no. 21, pp. 3729-3754, 1993.

[26] P.-S. Lee and K.-J. Bathe, "The quadratic mitc plate and mitc shell elements in plate bending," Advances in Engineering Software, vol. 41, no. 5, pp. 712-728, 2010.

[27] A. Laulusa, O. A. Bauchau, J.-Y. Choi, V. B. C. Tan, and L. Li, "Evaluation of some shear deformable shell elements," International Journal of Solids and Structures, vol. 43, no. 17, pp. 5033-5054, 2006. 


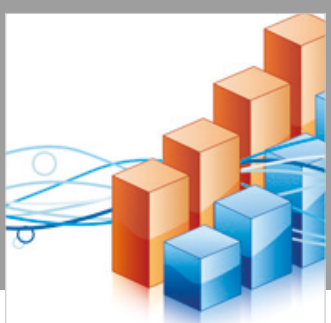

Advances in

Operations Research

\section{-n-m}

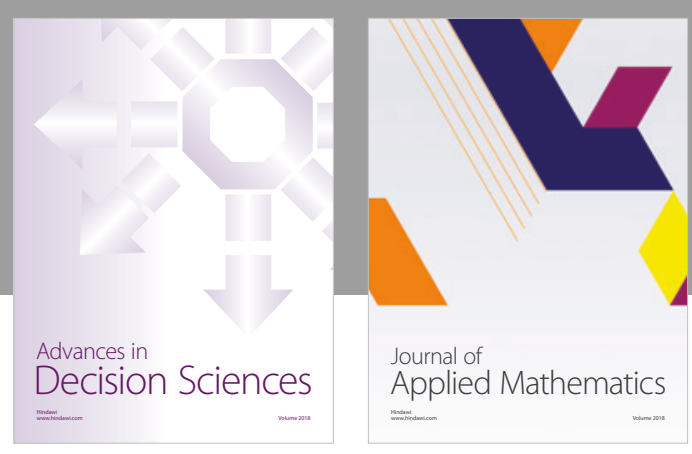

Journal of

Applied Mathematics
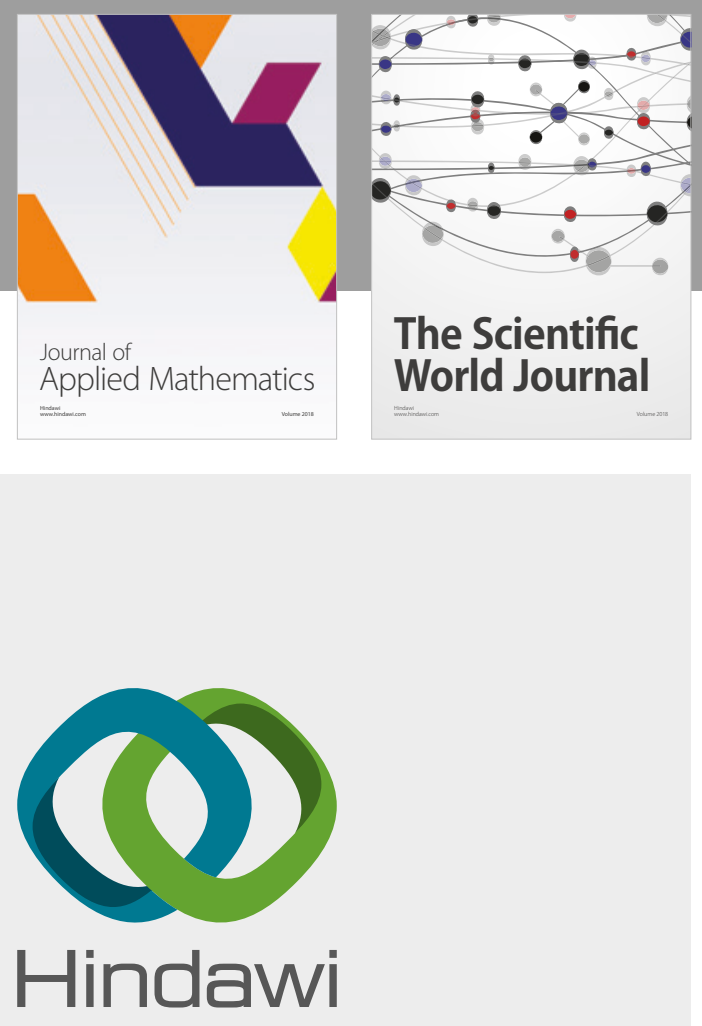

Submit your manuscripts at

www.hindawi.com

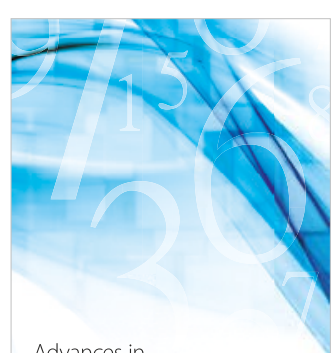

Advances in
Numerical Analysis
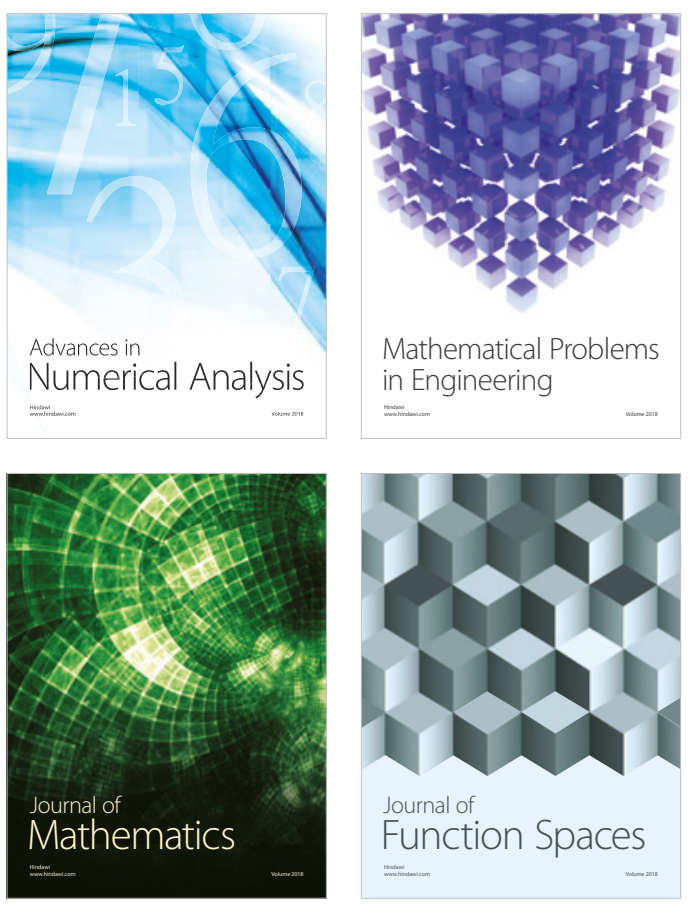

Mathematical Problems in Engineering

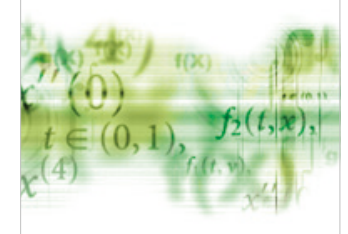

International Journal of

Differential Equations

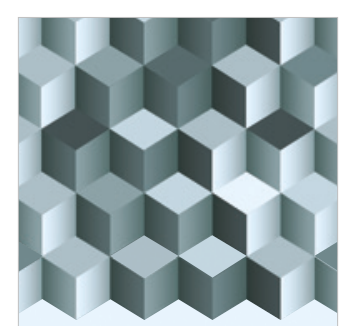

Journal of

Function Spaces
The Scientific

World Journal

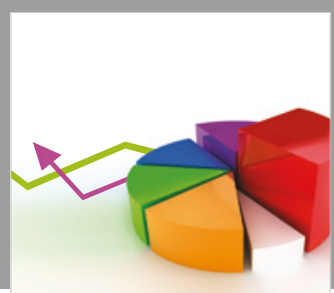

Journal of

Probability and Statistics
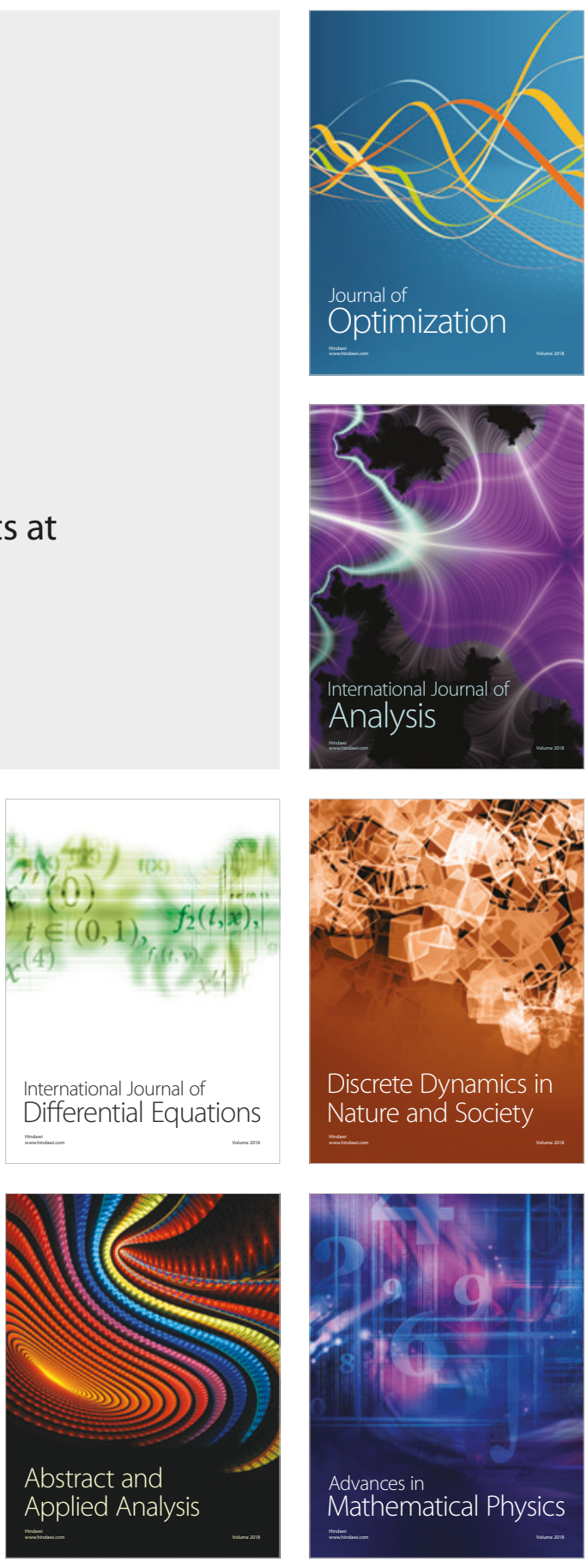